\title{
UMANO, SOVRUMANO O DIVINO? \\ LE DIVINITA' AUGUSTE E L'IMPERATORE A ROMA \\ di SILVIO PANCIERA
}

Quanto di umano, quanto di sovrumano e quanto di divino c'è nell' autorappresentazione del principe? E quanto di questa autorappresentazione combacia con l'immagine che i cittadini ne hanno? Inoltre quali sono i modi in cui, da varia parte, si contribuisce a costituire ed a mantenere un immaginario collettivo in cui la figura del principe tutto potrà comunque essere meno che quella di un uomo qualsiasi?

Le valutazioni degli studiosi non sono concordi, se è vero che anche nella bibliografia più recente continua a registrarsi una forte contrapposizione tra chi nega che vi sia mai stata una divinizzazione ufficiale dell'imperatore vivente ${ }^{1} \mathrm{e}$ chi invece la ritiene documentata, anche in Occidente, fin da Augusto. ${ }^{2}$

Mantenendomi ai margini del grande problema del cosiddetto culto imperiale $^{3}$ vorrei qui limitarmi a considerare la questione delle divinità auguste. Se le libazioni al Genio imperiale nei banchetti pubblici, l'inserimento dello stesso nella formula del giuramento e l'associazione del suo culto con quello dei Lari nei compita comportarono il trasferimento sul piano collettivo di pratiche religiose esistenti anche prima in ambito privato, e se il culto del numen dell'imperatore poté sembrare il punto estremo cui si poteva giungere senza varcare quella soglia di una dichiarata divinizzazione del sovrano che il costume romano rifiutava, ${ }^{4}$ quale significato vengono ad avere, nel quadro, le divinità auguste ed il loro culto?

\footnotetext{
' D. Fishwick, The Imperial Cult in the Latin West. Studies in the Ruler Cult of the Westem Provinces of the Roman Empire, II, 1 (Leiden 1991).

${ }^{2}$ M. Clauss, Kaiser und Gott. Herrscherkult im römischen Reich (Stuttgart und Leipzig 1999).

${ }^{3}$ Sul valore puramente convenzionale da attribuire a questa definizione si veda, da ultimo, J. Scheid, 'Honorer le prince et vénérer les dieux: culte public, cultes de quartier et culte impérial dans la Rome augustéenne, in Rome, les Césars et la Ville aux deux premiers siècles de notre ère (Rennes 2001), 85105.

${ }^{4} \mathrm{Su}$ ciascuno di questi punti, si vedano (con altra ampia bibliografia) i tre studi citati nelle note precedenti. Sul culto pubblico del Genius Augusti già dall'età augustea si veda in particolare, da ultimo: C. Letta, 'Il culto pubblico dei Lares Augusti e del Genius Augusti in una dedica metrica da Acerrae', Rivista di Cultura Classica e Medioevale 44 (2002), 35-43; Idem, 'Novità epigrafiche sul culto del Genius Augusti in Italia', Usi e abusi epigrafici. Colloquio Intermazionale di Epigrafia Borghesi 2001. Per la nuova lettura delle iscrizioni dell'arco di Medinaceli rispettivamente come: Numini Augusto sacru [m] (lato settentrionale) e Numini Imp(eratoris) Domitiani Aug(usti) Ger(manici), poi con Traiani al posto di Domitiani (lato meridionale): G. Alfoldy \& J.M. Abascal, El arco romano de Medinaceli (Madrid 2002), 71-103.
} 
È noto che dall'età augustea si moltiplicano i casi di divinità che assumono l'epiteto sostanzialmente, mai prima portato dalle stesse, di Augustus / Augusta. ${ }^{5}$ Nessuno ha dubitato che questo fenomeno sia da porre in relazione con l'inserimento nel 27 a.C. dello stesso epiteto, in funzione di cognomen, nella nuova denominazione progressivamente elaborata per il Principe. Si è discusso e si discute invece sul significato di questa novità dal punto di vista religioso. Può essere utile ricordare brevemente, sia pure correndo qualche rischio di schematizzazione eccessiva, le quattro principali posizioni assunte dagli studiosi al riguardo: ${ }^{6}$

1) Una dedica a Mercurio Augusto non esprime culto a questo dio, ma culto ad Augusto, o comunque all'imperatore in veste (ad esempio di generatore di ricchezza) propria anche del dio.

2) Mercurius Augustus non è l'imperatore in veste del dio evocato, ma il dio stesso, però legato con il Principe e con la sua famiglia in tale particolare unione da esserne guida, patrono, protettore in ogni circostanza e da essere quindi particolarmente idoneo ad ogni forma di venerazione rivolta a vantaggio dell'imperatore e della sua famiglia. Tale legame può essere generato, o accentuato, da motivi topici (ad esempio dalla connessione tra il tempio di Apollo e la domus imperiale sul Palatino, nel caso di Apollo Augustus).

3) Una dedica Mercurio Augusto può essere considerata equivalente a Mercurio et Augusto, vale a dire ad una dedica congiunta, espressa per asindeto, a Mercurio ed all'imperatore-dio.

4) Una dedica Mercurio Augusto deve esser vista come niente più che un atto in cui si fondono devozione e piaggeria: devozione per il dio e piaggeria per l'imperatore che, tramite l'epiteto, si vede a lui associato.

Come si vede, anche su questo particolare aspetto della più ampia questione del "culto imperiale", le posizioni sono molto divergenti: alcune implicanti vere forme di culto, dirette od oblique, dell'imperatore; altre distinguenti la figura del dio da quella del sovrano, e ciò ai più diversi livelli, da quello di una distinzione che non esclude un rapporto strettissimo e privilegiato, a quello della più disincantata negazione di un qualsiasi rapporto.

Colpisce, scorrendo la bibliografia disponibile, la mancanza di una puntuale verifica nelle fonti disponibili (ovviamente soprattutto epigrafiche) del reale stato delle cose nelle varie epoche e nei vari luoghi. In un libro edito

\footnotetext{
${ }^{5}$ Unica eccezione epigrafica: CLL V $4087=\mathrm{I}^{2} 753$ cfr. p. $946=\mathbb{I L L R P} 200$ (ager Mantuanus, a. 59 a.C.) [A]ug(ustis) Laribus.

${ }^{6} \mathrm{La}$ bibliografia al riguardo è sterminata; la principale si trova raccolta, oltre che nelle opere citate alle $\mathrm{nn} .1$ e 2, in S. Montero - S. Morea (cur.), Romana religio, religio Romanorum. Diccionario bibliográfico de religión romana (Madrid 1999), 136-140.
} 
da poco si fornisce, è vero, un elenco di ben 209 divinità cui risulta attribuito l'epiteto di Augustus / Augusta, ma senza una raccolta delle testimonianze pertinenti (viene data una sola citazione per divinità) e quindi senza alcuna possibilità di ripartizione areale e cronologica del culto delle medesime. ${ }^{7}$ Ipotesi interpretative e generali a parte, è evidente che quello di cui abbiamo bisogno per valutare l'estensione, l'importanza ed il significato del fenomeno, è invece di sapere quando, dove, a chi e da chi, il culto sia stato tributato. Per quanto mi consta, un'inchiesta del genere è stata fatta sistematicamente soltanto per la Spagna da Robert Étienne quasi cinquant'anni fa nel suo noto libro sul culto imperiale nella penisola iberica. ${ }^{8}$ Meno utilizzabile ai nostri fini un altro libro recente sulle province germaniche e sulla Gallia Belgica. ${ }^{9} \dot{\mathrm{E}}$ merito dell'Étienne aver portato la questione fuori dal generico con tutta una serie di osservazioni (sulla ripartizione geografica e cronologica delle iscrizioni, sull'importanza relativa degli dei in causa, sull'origine sociale dei fedeli e sul significato religioso e politico di questo tipo di culto), che attendono ancora di essere messe a confronto con quelle conseguibili applicando lo stesso metodo, in aree diverse. In questa circostanza, vorrei concentrare l'attenzione su Roma, che ha il vantaggio di costituire un ambito ben definito, di disporre, da sola, di una documentazione comparabile a quella di tutta la penisola iberica e, infine, fatto non trascurabile, di essere il centro stesso da cui il fenomeno del culto delle divinità auguste si può ritenere abbia preso le mosse, per di più nella immediata vicinanza e quindi sotto l'influsso ed il controllo più diretti del potere imperiale.

Tolti alcuni casi per vari motivi, ${ }^{10} \mathrm{i}$ documenti su cui si può contare per un'inchiesta sul culto delle divinità auguste a Roma, cioè le iscrizioni

\footnotetext{
${ }^{7}$ Clauss 1999, op. cit. (n. 2), 527-532.

${ }^{8} \mathrm{R}$. Étienne, Le culte impérial dans la Péninsule Ibérique d'Auguste à Dioclétian (Paris 1958), 319-349.

${ }^{9}$ U.-M. Liertz, Kult und Kaiser. Studien zu Kaiserkult und Kaiserverehrung in den germanischen Provinzen und in Gallia Belgica zur römischen Kaiserzeit (Rom 1998), in part. 163-188. Secondo M.-Th. Raepsaet Charlier, Diis deabusque sacrum (Paris 1993), 19, l'epiteto Augustus/Augusta si trova attribuito a divinità: 31 volte in Aquitania, 86 nella Lionese, 4 in Belgica, 2 nella Germania Inferiore e 22 nella Germania Superiore. Non considera le dediche alle divinità auguste A. Chastagnol, 'L'expression épigraphique du culte impérial dans les provinces gauloises', Revue des Études Anciennes 97 (1995), 593-614 (per la Narbonese vedi anche Idem in Revue Archéologiques de Narbonnaise 32 [1999], 59).

${ }^{10}$ Di dubbia autenticità o comunque di incerta interpretazione: CIL VI 437; cfr. A. Gunnella, AA.VV., Collezioni fiorentine di antichità, II, Palazzo Peruzzi, Palazzo Rinuccini (Roma 1980), $112 \mathrm{nr} .81$, tav. XXXII, 2-3: I(ovi) S(ancto) Aug(usto)? CIL; I(mperio) S(ilvani) Aug(usti)? Gunnella; vedi anche E. Schraudolph, Römischen Götterweihungen mit Reliefschmuck aus Italien. Altäre, Basen und Reliefs (Heidelberg 1993), 171 S4, tav. 14. - CIL VI 795: Eventui / Aug(usto) / feliciter. / Victoriis Aug(ustis) feliciter. La tradizione ha però Adventui e l'iscrizione mi sembra più acclamatoria per un un vittorioso ritorno imperiale che una dedica sacra. Cosi già H.L. Axtell, The Deification of Abstract Ideas in Roman Literature and Inscriptions (Chicago 1907), 52; vedi anche T. Holscher, Victoria Romana (Mainz am
} 
Rhein 1967), 63 (che parla tuttavia di ara) .- CIL VI 807. Dubbio se si riferisca a divinità e, nel caso, a quale: [Dia]nae, [Fortu]nae o [Fausti]nae, [Ploti]nae, Sabi[nae] Aug(ustae), CIL. Chi pone l'iscrizione, forse un liberto, sembra esser stato funzionario di un collegio. - CIL VI 30956: [L]aribus A[ug(ustis) - - ] / vici Bo[---]. Incerta l'appartenenza a Roma. Il Visconti la dice di Bovillae, il Dessau invece l'attribuisce congetturalmente a Roma, dove peraltro un vicus Bo[---] è altrimenti sconosciuto. - CLL VI 40615 (= 39426?) (lettura e integrazione della prima riga molto dubbie): [--] Aug(ust-) In[victo Soli?]; resterebbe comunque ignota la divinità augusta cui la dedica sarebbe stata posta pro salute et victoria et reditu di Settimio Severo, Caracalla e Geta l'1 gennaio 200 d.C.

L'epiteto è riferito a divinità, $\mathrm{ma}$ in contesto diverso da vere e proprie iscrizioni dedicatorie: $\mathrm{J}$. Scheid, Commentarii Fratrum Arvalium qui supersunt (Roma 1998), 285, fr. 99b, r. 2 cfr. $288 \mathrm{~s}$. Registrazione negli Atti degli Arvali di una serie di sacrifici verosimilmente in Capitolio, comprendente quello di una bos femina a Felicitas Augusta. - Scheid 1998, op. cit., 59, I, r. 17: Salus Augusta p(ublica) $p($ opuli $) Q($ uiritium $)$ figura tra le divinità invocate dei voti arvalici del 3 gennaio 91 d.C. per Domiziano e Domizia.

L'epiteto non compare in dediche a divinità, ma nella denominazione di are o signa (questi documenti saranno comunque eventualmente richiamati anche nelle note di commento alla lista A): $\mathrm{CIL}$ VI 130 cfr. pp. 3003, 3755. Supplementa Italica, Imagines, Roma 1 (Roma 1999), $380 \mathrm{nr} .1206$ : apparterrà probabilmente alla base di un'immagine di Diana Augusta posta il 19 ottobre 241 nelle adiacenze dei castra praetoria dai venatores immunes e dal custos vivarii cohortium praetoriarum et urbanarum. - Sacrifici ad aram Pacis Augustae sono ricordati tre volte negli Atti degli Arvali del tempo di Caligola (Scheid 1998, op.cit., 12b, r. 10, 12c r. 68, 15c, r. 10); l'epiteto di Augusta non è però attribuito a Pax in occasione di un sacrificio degli Arvali sul Campidoglio nel 66 (Scheid, ibidem, 30, I cd, r. 12). Per un aggiomamento problematico e bibliografico su quest'ara: Lexicon Topographicum Urbis Romae (d'ora in avanti LTUR) IV (Roma 1999), 70-74 e V (Roma 1999), 285 s. - Sacrifici ad aram Providentiae Augustae sono registrati due volte negli Atti del tempo di Caligola (Scheid 1998, op.cit., 12c, r. 56, 13f, r. 5). Su quest'ara, la sua storia e il suo significato, oltre a Scheid, cit., 285: LTUR IV (Roma 1999), 165 s. - CIL VI 632; ILS 5084 a. P. Sabbatini Tumolesi, Epigrafia anfiteatrale dell'Occidente Romano, I, Roma (Roma 1988), $55 \mathrm{nr}$. 46, tav. XV, fig. 2: si restaura un Silvanum Augustum, ciò̀ un signum del dio, per conto di un collegio di gladiatori denominati qualche anno prima, al momento della fondazione (CIL VI 631, a. 177 d.C.) Silvani Aureliani; 185-192 perché Commodo vi è menzionato con il titolo di Felix. - CIL VI $3721=31055$ : Inventori lucis Soli / invicto Augusto. Non è dedica ma legenda di un medaglione che, considerata falsa da F. Cumont, Textes et monuments figurés relatifs aux mystères de Mithra, II (Bruxelles 1896), $108 \mathrm{nr}$. 89, è invece ritenuta autentica da M. Guarducci, Rendiconti Pontificia Accademia Romana di Archeologia 30-31 (1957-1959), 161-169; vedi anche J. Ruysschaert, ibid. 37 (1964-1965), 325-336; L. Michelini Tocci, I medaglioni romani e $i$ contorniati del Medagliere Vaticano (Città del Vaticano 1965), 244. - CIL VI 103 cfr. p. $3003=30962$, cfr. p. 3755; ILS 1879: una Victoria Augusta, cioè un'immagine della dea verosimilmente accompagnata da un'iscrizione in cui era contenuto tale epiteto, figura tra i doni offerti alla schola degli scribi librari e dei preconi degli edili curuli nel Foro Romano, in occasione di un restauro databile tra i Giulio-Claudi e la prima età flavia (curatore del medesimo è un Bebryx Aug.l. Drusianus che prima di diventare liberto imperiale era stato schiavo, o del Druso morto nel 23 o del Druso morto nel 33: $\mathrm{H}$. Chantraine, Freigelassene und Sklaven im Dienst der römischer Kaiser [Wiesbaden 1967], $311 \mathrm{nr}$. 119, 361 s.). Dalle immediate vicinanze viene CIL VI 198 cfr. 30712, 36747: Victoriae Imp. Caesaris Vespasiani Augusti.

L'epiteto di Augustus non è attribuito ad una specifica divinità. CIL VI 540: Numini deor(um) Aug(ustorum) sacrum, verosimilmente da riferire al complesso delle divinità auguste, vedi Fishwick 1991, op. cit. (n. 1), 450-452 (diverse ipotesi in CIL). - CIL, VI 1201, 31260 cfr. pp. 846: Aug(---) sacr(-). Titulus Diis Augustis, non imperatori Augusto dedicatum esse puto: Alföldy, CIL VI p. 4335, con datazione al II sec. d.C. - CIL VI 30984 cfr. p. 3758. ILS 373 (III sec. d.C.): Numinibus / Aug(ustis), / Tutelae opti/mae .... 
dedicatorie, sono in tutto $90^{11}$ che elenco qui sotto nella lista A. I nomi degli dei (con relativo epiteto) vi figurano in ordine alfabetico nella forma che hanno nelle iscrizioni il cui riferimento bibliografico (CIL o dopo CIL) è dato di seguito. Segue la datazione. In nota fornisco, per ciascuna, altra bibliografia rilevante e gli elementi su cui si fonda la datazione che, per fortuna, può essere nella maggioranza dei casi, molto o abbastanza precisa.

A - Secondo divinità

\begin{tabular}{|c|c|c|c|}
\hline Nr. Id. & Divinità & Bibliografia & Datazione \\
\hline$\overline{\mathrm{A} 1}$ & Aiscolapio Augusto & 12 cfr. 30684, pp. $3003.3753^{12}$ & 25 d.C. \\
\hline A2 & Aesculapio Aug. & 30983 cfr. p. $3758^{13}$ & Età adrianea? \\
\hline A3 & Apollini Aug. & $33^{14}$ & 2/1 a.C. \\
\hline A4 & Apollini Aug. & 35 cfr. p. $3755^{15}$ & 46 d.C. \\
\hline A5 & Apollini Aug. & $42^{16}$ & 115 d.C. \\
\hline A6 & [Conclordliael Aug. & $A E 1971,27^{17}$ & età auqustea \\
\hline A7 & [Concjord[iae] Aug. & $A E 1913,1^{18}$ & | sec., I metà \\
\hline A8 & [D]ianae August. & 128 cfr. pp. $3003.3755^{19}$ & 7/6 a.C. \\
\hline
\end{tabular}

\footnotetext{
${ }^{11}$ Solo latine; nessuna attestazione nelle IGUR.

${ }^{12}$ ILS 3837. K.P. Almar, Inscriptiones Latinae (Odense 1990), 145 nr. 99 con foto. Ara marmorea dall'Isola Tiberina, forse dal compitum del vicus Censori; LTUR V (Roma 1999), 157. La data si ricava dall'anno $31^{\circ}$ dell'era compitale.

${ }^{13}$ ILS 3840. Leggo: Aesculapio et Saluti Aug(ustis), riferendo l'epiteto ad entrambe le divinità e non solo alla seconda. Lastra dagli Horrea Galbana: LTUR III (Roma 1996), 40 ss. Errata la datazione all'età di Galba proposta in Revue des Études Anciennes 86 (1984), 228; per la presenza tra i dedicanti di Ulpii e P. Aelii sarà almeno di età adrianea o posteriore.

${ }_{14}$ Base del compitum di via Marmorata, sul quale: LTUR IV (Roma 1999), 261 s. Qui e altrove l'iscrizione è erroneamente datata all'l d.C.; i magistri vici che la dedicano sono in realtà quelli del $2 / 1$ a.C. come risulta dall'anno dell'era compitale (il sesto) e dai fasti del compitum: I.It., XIII, 1 p. 285.

${ }^{15}$ ILS 3219. Ara dal compitum di via Marmorata (vedi n. prec.). La datazione deriva dall'anno $52^{\circ}$ dell'era compitale.

${ }^{16}$ ILS 1634. Base dalla Moneta: LTUR III (Roma 1996), 280 ss. Per la data cfr. CIL VI 43 (A14), 44 (A20) del 28 gennaio (115 d.C.) dies imperii di Traiano, e 239 (Genio fam. monetal.); vedi anche A25 ed A32: P. Herz, Untersuchungen zum Festkalender der römischen Kaiserzeit nach datierten Weih- und Ehreninschriften (Mainz 1975), 136.

${ }^{17}$ A.M. Colini, Rendiconti della Pontificia Accademia Romana di Archeologia 43 (1970/71), 55-70 con foto e ricostruzione grafica. Ara da compitum in piazza Bocca della Verità. La datazione in età augustea, preferibilmente nel periodo 12-7 a.C., è suggerita all'editore dal contesto archeologico, dalla tipologia dell'ara in travertino, dalla divinità cui è dedicata, dalla corona civica che vi è rappresentata e da considerazioni storiche.

${ }^{18}$ H.L. Wilson, American Joumal of Archaeologgy 16 (1912), 94-96 con fig. 1 (AE 1913, 1); ILS 9517; H. Gummerus, Klio 14 (1914), 145 s.; J. Andreau, La vie financière dans le monde romain (Rome 1987), 681 s. R. Friggeri, La collezione epigrafica del Museo Nazionale Romano delle Terme di Diocleziano (Roma 2001), 74 fig. 7. Lastra che si asserisce trovata fra il Tevere ed il Monte Testaccio. La datazione comunque nella prima età imperiale è suggerita dalla divinità, dalla paleografia e dal fatto che i dedicanti offrono, oltre ad una statua della dea, tre imagines, forse d'argento, verosimilmente riferibili a personaggi della casa imperiale. I pigmentarii ed i miniarii attestati nell' iscrizione sono sicuramente presenti a Roma già in età augustea: C. Ricci, Gerión 10 (1992), 129.
} 


\begin{tabular}{|c|c|c|c|}
\hline Nr. Id. & Divinità & Bibliografia & Datazione \\
\hline$\overline{A 9}$ & Dianae Augustae & $129^{20}$ & 1 a.C./1 d.C. \\
\hline A10 & Dianae Augustae & $36864^{21}$ & età augustea? \\
\hline A11 & [Di]anae Aug. & $36868^{22}$ & II sec., II metà \\
\hline A12 & Fonti Aug. & $150^{23}$ & 244-249 d.C. \\
\hline A13 & Fort[unae] Aug. & $36773^{24}$ & $1 / 11 \mathrm{sec}$. \\
\hline A14 & Fortunae Aug. & 43 cfr. p. $3755^{25}$ & 115 d.C. \\
\hline A15 & $\begin{array}{l}\text { Fortunae Augustae } \\
\text { Respicie[nti] }\end{array}$ & $181^{26}$ & II sec. fine/lll sec \\
\hline A16 & $\begin{array}{l}\text { Fortunae Augustae } \\
\text { Praesenti }\end{array}$ & $181 b^{27}$ & II sec. fine/lll sec \\
\hline A17 & [He]rculi Tuta[tori A]ug. & 343 cfr. 30743 , pp. $833.3004^{28}$ & 26 d.C. \\
\hline A18 & Herculi Aug. & 301 cfr. 30731 , pp. $3004.3756^{29}$ & età vespasianea \\
\hline A19 & Herculi Aug. & 299 cfr. p. $3004^{30}$ & $\mid 1 / 1 / \mathrm{sec}$. \\
\hline A20 & Herculi Aug. & 44 cfr. p. $3755^{31}$ & 115 d.C. \\
\hline
\end{tabular}

${ }^{19}$ Base $\mathrm{o}$ ara da compitum di ignota ubicazione. La data si ricava dall'anno $1^{\circ}$ dell'era compitale.

${ }^{20}$ Base $\mathrm{o}$ ara da compitum di ignota ubicazione. La data è ricavata dall'anno $7^{\circ}$ dell'era compitale. Tra i dedicanti compare un $M$. Lurius Maritimus per il quale vedi anche n. s.

${ }^{21}$ Ara dal Viminale. LTUR II (Roma 1995), 16. La datazione, indicata soltanto con giorno e mese (12 agosto) è suggerita sulla base della tipologia dell'ara e dello stile dei rilievi sui fianchi: $\mathrm{H}$. C. Bowerman, Roman Sacrificial Altars (Bryn Mawr 1913), 97; E. Schraudolph 1993, op. cit. (n. 10), 126 D5, tav. 2. Da notare che la dedica è fatta da un $M$. Lurius Saturninus e che un M. Lurius Maritimus compare tra i dedicanti di un'altra base o ara a Diana Augusta datata tra l'1 a.C. e l'1 d.C. (vedi n. prec.).

${ }^{22}$ Due frammenti di tavola ora riediti con un terzo (CIL VI 38398), di cui prima non era stata riconosciuta la pertinenza, in La collezione epigrafica dell'Antiquarium Comunale del Celio (Roma 2001), 368 s., nr. 409, con tav. LXIV, fig. 1. L'intestazione Silvano et Dianae Aug(ustis), ritenuta dubbia in CIL, non sembra da escludere dopo una verifica della parte conservata. La datazione è ricavata essenzialmente dalla paleografia e della qualifica di me(n)s(or) ae[dificiorum] Augg. (i. e. Augustorum). Per l'offerta di un signum di Diana Augusta nel 241 d.C., vedi sopra in n. 10.

${ }^{23}$ Parva basis dal Celio, d'incerto collegamento con la casa dei Filippi, in cui uno schiavo è autore della dedica: LTUR I (Roma 1993), 209; II (Roma 1995), 156; vedi anche G. Cressedi, Un manoscritto derivato dalle "Antichità" del Piranesi, Vaticano - Latino 8091 (Roma 1975), 169; L. Avetta, Roma - Via Imperiale (Roma 1985), 27 s.; E. Tortorici, Bullettino della Commissione Archeologica Comunale di Roma 95 (1993), 161-172. Incerto il significato delle tre litterae singulares (M. P. R.) che seguono la dedica Fonti Aug.. Si conosce in area un Fons Mercuri. Siccome però lo schiavo dice di aver rifatto la dedica, non escluderei che possano essere piuttosto le iniziali del nome del dedicante originario.

${ }^{24}$ Ara. Cercata invano nel Foro Romano, dove fu vista: Iscrizioni greche e latine del Foro Romano e del Palatino (Roma 1996), 56. La datazione dipende dal giudizio litteris pulchris del Bang.

${ }^{25} \mathrm{ILS}$ 1634. Base dalla Moneta (vedi sopra n. 16), esplicitamente datata al 28 gennaio 115, dies imperii di Traiano.

${ }^{26}$ ILS 3704. Basis (CIL). La data si ricava dal fatto che il dedicante è un M. Aur(elius) Ctesias Iunior. Sul tempio e il vicus della Fortuna Respiciens: LTUR II (Roma 1995), 276; V (Roma 1999), 166. La base fu vista in hortis Carpensibus in Quirinali, ma l'originaria collocazione poté essere tuttaltra.

${ }^{27} \mathrm{ILS} 3704$. Sulla stessa base del numero precedente.

${ }^{28}$ Base o ara da compitum incerto della Regio X: LTUR III (Roma 1999), 200. Datazione espressamente indicata con anno vicano e coppia consolare.

${ }^{29}$ Ara dall'Emporium (Marmorata): LTUR II (Roma 1995), 222; III (Roma 1996), 223. Il dedicante è uno schiavo di Vespasiano.

${ }^{30}$ Epistilio, cercato invano sul Palatino dove fu visto: Iscrizioni greche e latine, cit (n. 13), 39: LTUR III (Roma 1996), 12. La datazione è congetturale. 


\begin{tabular}{|l|l|l|l|}
\hline Nr. Id. & Divinità & Bibliografia & Datazione \\
\hline A21 & Herculi Aug. & 298 cfr. p. $3756^{32}$ & età traianea? \\
A22 & Herculi August. & AE $1927,145^{33}$ & età commodiana? \\
A23 & Ilsijdi Aug. & $349^{34}$ & II sec. \\
A24 & Iovi Maleciabrudi Aug. & $36792^{35}$ & III sec. \\
A25 & I.O.M.H. Aug. & 422 cfr. 30765, pp. $3005.3756^{36}$ & II sec., fine \\
A26 & Laribus Augustis & 445 cfr. p. $3756^{37}$ & $7 / 6$ a.C. \\
A27 & Larib. Aug. & 446 cfr. p. $3005.3756^{38}$ & $7 / 6$ a.C. \\
\hline
\end{tabular}

${ }^{31}$ ILS 1635. A. E. Gordon, Album of Dated Latin Inscriptions II (Berkeley- Los Angeles 1964), 40, nr. 175, tav. 77, figg. a, b; G. Spinola, Il Museo Pio Clementino II (Città del Vaticano 1999), 58, nr. 77, fig. 10. Ara dalla Moneta (vedi sopra n. 16). Datazione esplicita al 28 gennaio $115(119$, per errore $I L S$ e altri).

${ }^{32}$ ILS 1636. Ara o base di provenienza ignota, ma verosimilmente dalla Moneta come CIL VI 42 (A5), 43 (A16), 44 (A22), tutte del 115 d.C. La datazione in età traianea è proposta per confronto con queste.

${ }^{33}$ O. Marucchi, Rendiconti della Pontificia Accademia Romana di Archeologia 4 (1925/26), 394 fig. 5; per la lettura corretta collegio iuvenum Racilianensium (non Racillanensium) e per la provenienza dall'area dei vici Raciliani maioris e minoris in Trastevere, S. Panciera, Archeologia Classica 22 (1970), 159 ss.; LTUR IV (Roma 1999), 249; V (Roma 1999), 187. La datazione è ricavata dalla paleografia e dalle particolari relazioni con Ercole di Commodo.

${ }^{34}$ Frammento indefinibile di provenienza ignota. Datazione su base paleografica, vedi I. Di Stefano Manzella, Index Inscriptionum Musei Vaticani, I, Ambulacrum Iulianum sive "Galleria Lapidaria" (Roma 1995), $261 \mathrm{nr}$. 36. Il dedicante, il cui nome non è da leggere necessariamente [ - - ] Datus (altrettanto possibile è il comune Se]datus; vedi anche il meno comune Man]datus), sembra essere quaestor in una comunità isiaca. Nemmeno un'integrazione [Serapi]di può tuttavia essere esclusa. L. Vidman, Sylloge inscriptionum religionis Isiacae et Serapiacae (Berlin 1969), $207 \mathrm{nr} .399$ (con datazione I-II sec.); M. Malaise, Inventaire préliminaire des documents égyptiens découverts en Italie (Leiden 1972), $115 \mathrm{nr}$. 10; Idem, Les conditions de pénétration et de diffusion des cultes égyptiens en Italie (Leiden 1972), 145 nn. 2,10.

${ }^{35}$ ILS 9282. Ara del c.d. Santuario siriaco sul Gianicolo; LTUR III (Roma 1996), 138-143. La datazione si ricava principalmente dall'osservazione secondo cui l'iscrizione ne sostituisce una precedente dopo un incendio che ha distrutto il santuario della fine del II sec., (in coincidenza con un terremoto nel 242): F. Duthoy - J. Frel, 'Observations sur le sanctuaire syrien du Janicule', Orientalia sacra urbis Romae (Roma 1996), 296, 298.

${ }^{36}$ ILS 4292; Y. Hajjar, La Triade d'Héliopolis-Baalbek. Son culte et sa diffusion à travers les textes littéraires et les documents iconographiques (Leiden 1977) I, 357 ss., nr. 286; II, tav. CDX. Base conservata dal Medioevo alle pendici del Gianicolo, ma proveniente senza dubbio dal c.d. Santuario siriaco (vedi n. 35). Per la datazione alla fine del II sec. vedi già Chr. Hülsen, Römische Mitteilungen 22 (1907), 247 ed ora Duthoy - Frel 1996, op. cit. (n.35), 296; preferiscono il III sec. B. M. Felletti Maj, Bullettino della Commissione Archeologica Comunale di Roma 75 (1953-1955), 140 n. 11; N. Goodhue, The Lucus Furrinae and the Syrian Sanctuary on the Janiculum (Amsterdam 1975).

${ }^{37}$ ILS 3613. Spinola 1999, op. cit. (n. 31), 202 s., nr. 17, con principale bibliografia precedente. Ara di provenienza ignota. La datazione si ricava dall'anno $1^{\circ}$ dell'era compitale. Sullinsicurezza dell'integrazione Laribus Augustis G[enis Caesaru] $m$ data in CIL, vedi già Dessau in ILS. Altri: G[enio Caesaris] o G[enio Augusti] vedi E. Hänlein Schäfer, Subject and Ruler (Ann Arbor 1996), 76 con nn. $19,91,95$.

${ }^{38}$ ILS 3612; Museo Nazionale Romano. Le sculture I, 2 (Roma 1981), 70 s.; M. Hano, in W. Haase \& H. Temporini, edd., Aufstieg und Niedergang der Römischen Welt II, 16, 3 (Berlino/ New York 1986), 2342; Hänlein Schäfer 1996, op.cit. (n. 37), 95 nr. 6, sempre con altra bibliografia; Friggeri 2001, op.cit. (n. 18), 72, fig. 5. Ara dall'Isola Tiberina, verosimilmente dal compitum del vicus Censori: LTUR III (Roma 1996), 100. La data si ricava dall'anno $1^{\circ}$ dell'era compitale. 


\begin{tabular}{|l|l|l|l|}
\hline Nr. Id. & Divinità & Bibliografia & Datazione \\
\hline A28 & Larib. Aug. & 447 cfr. p. $3005.3756^{39}$ & $7 / 6$ a.C. \\
A29 & Laribu[s Aug.] & L'Urbs (Roma 1987$), 64-70^{40}$ & $7 / 6$ a.C. \\
A30 & Laribus Augustis & $448 \mathrm{cfr} . \mathrm{p} .3756^{41}$ & 2 a.C. \\
A31 & Laribus Augustis & $36809^{42}$ & 2 a.C. \\
A32 & Larib. August. & 30957 cfr. p. $3758^{43}$ & 2/3 d.C. \\
A33 & Lar. Aug. & AE1964, $77^{44}$ & $3 / 4$ d.C. \\
A34 & Lar. [Aug.] & AE 1948, $82^{45}$ & età augustea \\
A35 & Laribus Augusti[s] & 443 cfr. p. $3005^{46}$ & età tiberio-claudiana \\
A36 & Laribu[s Aug.] & AE1980, $55^{47}$ & I sec., I metà \\
A37 & Laribus Augustis & AE 1960, $6^{48}$ & 56 d.C. (?) \\
\hline
\end{tabular}

${ }^{39}$ ILS 3612a; Liebighaus Museum alter Plastik Frankfurt am Main. Führer durch die Sammlungen Antike Kunst (Frankfurt am Main 1980), 267 s.; M. Buonocore, Camillo Massimo collezionista (Roma 1996), 195, $200 \mathrm{nr}$. 87. Ara compitale trovata sull'Isola Tiberina con la precedente e datata allo stesso anno (vedi n. precedente).

${ }^{40}$ Omesso in AE. Epistilio dal compitum del vicus Aesculeti: LTUR I (Roma 1993), 316. La data si ricava dall'anno $1^{\circ}$ dell'era compitale.

${ }^{41}$ LLS 3614; Hänlein Schäfer 1996, op.cit. (n. 37), 95 nr. 2 con bibliografia precedente. Ara del compitum vici Sandaliari: LTUR I (Roma 1993), 57; V (Roma 1999), 189. La data è doppiamente espressa con l'era vicana e la coppia consolare.

${ }^{42}$ Sull'altro lato [La]rib. Aug. ILS 9250. Foto e bibliografia in Supplementa Italica. Imagines (1999), op. cit. (nt. 10), nr. 5. Ara del compitum del vicus Statae Matris. LTUR V (Roma 1999), 191. La datazione è indicata con coppia consolare e anno $6^{\circ}$ di era vicana.

${ }^{43} \mathrm{LS}$ 3615. Per una più corretta lettura dell' iscrizione sulla fronte: S. Panciera, in L'Urbs (Roma 1987), $68 \mathrm{~s}$. Foto e bibliografia in Supplementa Italica. Imagines (1999), op. cit. (n. 10), nr. 6. Ara compitale del vicus Aesculeti (vedi sopra n. 40). La datazione si ricava dall'anno $9^{\circ}$ dell'era compitale.

${ }^{44}$ A.M. Tamassia in Bullettino della Commissione Archeologica Comunale di Roma 78 (1961/2), $161 \mathrm{~s}$, figg. 34. Ara del compitum Acilium di cui è attestata l'esistenza ben prima della riorganizzazione augustea: M. Dondin - Payre, L'Urbs (Roma 1987), 87-109; LTUR I (Roma 1993), 314 ss.; D. Palombi, Tra Palatino ed Esquilino. Velia, Carinae, Fagutal (Roma 1997), 39-43; Idem, Rendiconti della Pontificia Accademia Romana di Archeologia 70 (1997-1998 [2000]), 115-135; F. Coarelli, Eutopia, n.s., 1 (2001), 7-43 (sulla topografia dell'area). La datazione si ricava dall'anno $10^{\circ}$ dell'era compitale, di cui ingiustamente dubita (controllo autoptico) Dondin - Payre, op.cit., 103 n. 24.

${ }^{45}$ C. Pietrangeli, Bullettino della Commissione Archelogica Comunale di Roma 70 (1942), 127-130 figg. 1-2; vedi anche W. Hermann, Römischen Götteraltäre (Kallmunz 1961), 89 s., nr. 19; Schraudolph 1993, op. cit. (n. 10), 230 L 98. Frammento di ara di provenienza ignota. Datazione su base stilistica e prosopografica.

${ }^{46}$ Ara di provenienza ignota. Datazione su base paleografica e stilistica. Schraudolph 1993, op.cit. (n. 10), 229 L 92 tav. 34.

${ }^{47}$ S. Panciera, Archeologia Laziale 3 (1980), 204 s., nr. 8, tav. XLIX, 1. Ara compitale dall'incrocio tra viale Africa (oggi Aventino) e via Aventina. La datazione si ricava dalla tipologia e dalla decorazione dell'ara.

${ }^{48}$ L. Moretti, Archeologia Classica 10 (1958), 231 s. nr. 1 (Idem, Tra epigrafia e storia [Roma 1990], 155 s. nr. 1); A.M. Colini - L. Cozza, Ludus Magnus (Roma 1962), 148 nr. 2, fig. 162; E.M. Smallwood, Historia 17 (1968), 384. Lastra dal compitum del vicus Cornicularius: LTUR V (Roma 1999), 160. La datazione è indicata, oltre che con il $61^{\circ}$ anno dell'era compitale, con la coppia consolare L. Iunio Gallione T. Cutio Cilto, già attribuita al 55 d.C., oltre che dal Moretti e dalla Smallwood, da P.A. Gallivan, Classical Quarterly 24 (1974), 293, 295, ma ora, con diverse argomentazionie, al 56 da G. Camodeca, Zeitschrift für Papyrologie und Epigraphik 63 (1986), 211-215, in part. 206-208. Sulla 


\begin{tabular}{|c|c|c|c|}
\hline Nr. Id. & Divinità & Bibliografia & Datazione \\
\hline A38 & Laribus Aug. & 449 cfr. p. $3756^{49}$ & 83 d.C. \\
\hline A39 & Larib[us Aug.] & $\begin{array}{l}\text { L'Urbs (Roma 1987), 70-73 fig. 6- } \\
7^{50}\end{array}$ & età domizianea \\
\hline A40 & Laribus Augustis & 450 cfr. 30768, pp. $3005.3756^{51}$ & $98 / 99$ d.C. \\
\hline A41 & La[ribus] A[ugustis] & $\begin{array}{l}\text { Bull. Com.,90, } 2 \text { (1985), } 279 \mathrm{nr} . \\
18^{52}\end{array}$ & I sec. \\
\hline A42 & Laribus Aug. & 30954 cfr. p. $3758^{53}$ & $\begin{array}{l}\text { I sec., || metà/ I| sec., I } \\
\text { metà }\end{array}$ \\
\hline A43 & [Laribu]s Aug. & 453 cfr. pp. $3005.3756^{54}$ & I sec., fine / II sec., in. \\
\hline A44 & Laribus Augustis & 451 cfr. 30769, pp. $3005.3756^{55}$ & 100 d.C. \\
\hline A45 & [Laribus A]ugustis & 452 cfr. pp. $3005 \cdot 3756^{56}$ & 109 d.C \\
\hline A46 & La[rum Aug.] & $30958^{57}$ & 116 d.C. \\
\hline A47 & [Laribus Aug]ustis & $A E 1971,33^{58}$ & 149 d.C. \\
\hline A48 & [Laribus Augusto]rum & $A E 1971,34^{59}$ & 161 d.C. \\
\hline
\end{tabular}

questione delle ere compitali anomale vedi da ultimo A. Fraschetti, Roma e il principe (Roma - Bari 1990), 265-268.

${ }^{49}$ ILS 3617; D. Modonesi, Museo Maffeiano. Iscrizioni e rilievi sacri latini (Roma 1995), 78 s. nr. 83 con foto. Epistilio dell'edicola compitale del vicus Honoris et Virtutis: LTUR V (Roma 1999), 167 s. La datazione si ricava dalla titolatura di Domiziano (cos. VIII, des. $X$ ) e dall'anno $9^{\circ}$ dell'era compitale.

${ }^{50}$ Omesso in AE. Frammento di architrave da rifacimento dell'edicola compitale del vicus Aesculeti (vedi sopra n. 40). Ė presente il nome di Domiziano; il terminus ad quem è costituito dall' incendio dell' 80 .

${ }^{51}$ ILS 3618. Epistilio dall'edicola compitale del vicus Portae Collinae: LTUR III (Roma 1996) 326; IV (Roma 1999) 181, 198, 316. La data si ricava dalla titolatura di Traiano (cos. II).

${ }_{52}$ Base o ara di provenienza ignota, forse da un vicus [---] Secundi, come proposto dall'Editrice (M.G. Granino Cecere) per confronto con il vicus Longi Aquilae. La datazione è su base paleografica.

${ }^{53}$ Ara dal Foro Romano, dove si conserva (inv. 12489). Discusso un eventuale rapporto con l'aedes Larum in summa Sacra via; favorevole F. Coarelli, in LTUR III (Roma 1996), 174; per altri piuttosto da mettere in rapporto con un compitum. Datazione su base essenzialmente paleografica. P. Zanker, Bullettino della Commissione Archeologica Comunale di Roma 82 (1970/71), 149 n. 8, nr. 11 esclude che appartenga alla serie degli altari augustei. Nessuna proposta da parte di M. Hano 1986, op.cit. (n. 38), 2343 s., tav. III, 5; Schraudolph 1993, op.cit. (n. 10), 229 L 95; Hänlein Schäfer 1996, op.cit. (n. 37), $96 \mathrm{nr} .8$.

${ }^{54}$ ILS 3616. Foto in Supplementa Italica. Imagines 1999, op. cit. (n. 10), nr. 129. Ara compitale del vicus Trium ararum: LTUR V (Roma 1999) 195. Datazione su base paleografica e prosopografica; sulla pretura di C. Pontius Faustinus Granianus PIR ${ }^{2}$ P 799; non posteriore al 136 essendo ancora in funzione la commissione augustea di sorveglianza sulle Regiones: S. Panciera, Archeologia Classica 22 (1970), $146-151$

${ }^{55}$ ILS 3619. Foto e bibliografia in Supplementa Italica. Imagines 1999, op.cit. (n. 10), nr. 2174. Epistilio dall'Isola Tiberina, verosimilmente dal compitum del vicus Censori (vedi sopra nn. 38, 39). La datazione è espressa con la coppia consolare e con l'anno dell'era compitale, però verosimilmente incompleto.

${ }^{56}$ ILS 3620. Foto e bibliografia in Supplementa Italica. Imagines 1999, op.cit. (n. 10), nr. 2175. Epistilio dall'edicola del compitum del vicus Iovis Fagutalis: LTUR III (Roma 1996), 135, 193. La datazione si ricava dalla titolatura di Traiano e corrisponde all'anno $121^{\circ}$ (non si sa sé da correggere) dell'era compitale.

${ }^{57}$ Cfr. F. Bianchi \& P.L. Tucci, Mélanges de l'École Française de Rome, Antiquité 108 (1996), 47-53. Epistilio di edicola da un compitum di Trastevere. La datazione si ricava dalla titolatura di Traiano.

${ }^{58}$ S. Panciera, Archeologia Classica 22 (1970), 138-151 con foto e ricostruzione grafica (parte delle osservazioni in $A E$ sono infondate). Lastra pertinente ad un'edicola compitale ignota. La data si ricava dalla titolatura di Antonino Pio e dalla coppia consolare. 


\begin{tabular}{|l|l|l|l|}
\hline Nr. Id. & Divinità & Bibliografia & Datazione \\
\hline A49 & [Larib]us Augg. & $455^{60}$ & 168 d.C. \\
A50 & Laribus Aug. & $454^{61}$ & II sec. \\
A51 & Larib. Aug. & $30959^{62}$ & 203 d.C. \\
A52 & Laribus Aug. & 30960 cfr. p. $3758^{63}$ & 223 d.C. \\
A53 & [La]ribus Alugustis] & $30961^{64}$ & $222-235$ d.C. \\
A54 & Laribus Aug. & $441^{65}$ & $?$ \\
A55 & Larib. Aug. & $442^{66}$ & $?$ \\
A56 & Laribus Augustis & $444^{67}$ & $?$ \\
A57 & Laribus Aug. & $30952^{68}$ & $?$ \\
A58 & [La]ribus Augustis & $3701=30962^{69}$ & $?$ \\
A59 & Mar[ti] Aug. & $483^{70}$ & I sec., I metà? \\
A60 & Marti Aug. & $484^{71}$ & I /l sec. $?$ \\
A61 & Mercurio Augus[to] & 283 cfr. p. $3004^{72}$ & $7 / 6$ a.C. \\
\hline
\end{tabular}

${ }^{59}$ L. Berni Brizio, Rendiconti dell'Accademia Nazionale dei Lincei, cl. mor., ser. VIII, 26 (1971), 769778. Architrave di edicola compitale del [vicus statu]ae Verris sulla cui discussa ubicazione si veda, oltre alla prima editrice (Regio LX), R.E.A. Palmer, Rendiconti della Pontificia Accademia Romana di Archeologia 51-52 (1978-1980), 111-136 (Regio IV); vedi anche F. Castagnoli, Rivistai di Filologia e Istruzione Classica 90 (1982), 499 n. 5 e Palombi, 1997, op.cit. (n. 44), 122 n. 195. La datazione si ricava dalla titolatura di Marco Aurelio e Lucio Vero.

${ }^{60}$ Nuovo lettura e interpretazione: A. Kolb, Zeitschrift für Papyrologie und Epigraphik 107 (1995), 205211 con foto (AE 1995, 91). Frammenti di tavola di provenienza ignota. Datazione consolare.

${ }^{61}$ Ara del chiostro di S. Paolo f.l.m. campata XIX, 6: G. Filippi, Indice della raccolta epigrafica di S. Paolo fuori le mura (Città del Vaticano 1999), $40 \mathrm{nr}$. 51339. Tra i magistri c'è un Ulpius.

${ }^{62}$ Frammento di tavola da S. Martino ai Monti. Datazione dalla titolatura imperiale. Mi chiedo se parte di questa iscrizione, non più esistente nell'Antiquarium comunale del Celio, sia da riconoscere in alcuni frammenti recentemente pubblicati come inediti in CIL VI 40605 e 40750 e visti nelle casse già al Palazzo delle Esposizioni contenenti appunto materiali sgombrati dall'Antiquarium.

${ }^{63}$ ILS 3621. Il genitivo del nome imperiale non dipende da Genio, ma da permissu: S. Panciera, Archeologia Classica 22 (1970), 148 s.. Tavola da edicola compitale del vicus Vestae: LTUR V (Roma 1999), 198. Datazione consolare

${ }^{64}$ Per il genitivo del nome imperiale vedi n. precedente. Foto e bibliografia in Supplementa Italica. Imagines, 1999, op. cit. (n. 10), nr. 1209. Frammento di tavola proveniente da edicola compitale di vicus ignoto. Datata dal nome di Severo Alessandro.

${ }^{65}$ Ara o base di provenienza ignota. Perduta. Non databile.

${ }^{66}$ Ara o base d'ignota provenienza. Si conserva ancora nelle Grotte Vaticane.

${ }^{67}$ LS 7280. Epistilio (?) visto alle pendici del Palatino verso S. Gregorio. Perduto. Non databile.

${ }^{68}$ Aretta pulvinata da area tra Ponte Sisto e Farnesina (Trastevere): LTUR V (Roma 1999), 167. Si conserva ancora al Museo delle Terme, inv. 27253. Il CIL, la dice litteris recentioribus.

${ }^{69}$ Epistilio dalle vicinanze di SS. Giovanni e Paolo nei cui pressi si trovava il compitum del vicus Trium ararum, vedi sopra n. 54. Perduto. Non databile.

${ }^{70}$ Base o ara trovata sub Aventino in ripa Tiberis. Perduta. La datazione è proposta per un possibile rapporto tra il dedicante (un M. Lollius) e i non lontani Horrea Lolliana prima del loro passaggio nella proprietà imperiale con Claudio (E. Rodriguez Almeida, Il Monte Testaccio (Roma 1984), 101 n. 5. Sugli horrea vedi anche LTUR III (Roma 1996), $43 \mathrm{~s}$.

${ }^{71}$ Base o ara di provenienza ignota. Perduta. Il dedicante (T. Tettius Pudes) è veterano di un'unità non indicata, forse urbana.

${ }^{72}$ Sembrerebbe ara o base (ma Fabretti la dice in epistylio). Certo da un compitum nei pressi di S. Maria in Cosmedin di dove deve venire anche A 86: S. Panciera, Archeologia Laziale 3 (1980), 203-206. Datazione sulla base dell'anno $1^{\circ}$ dell'era compitale. 


\begin{tabular}{|c|c|c|c|}
\hline Nr. Id. & Divinità & Bibliografia & Datazione \\
\hline$\overline{\mathrm{A} 62}$ & Mercurio Aug. & $34^{13}$ & $3 / 2$ a.C. \\
\hline A63 & Mercurio Aug. & $A E 1914,137^{74}$ & età augustea \\
\hline A64 & Merc. Aug. & $519^{75}$ & I sec.? \\
\hline A65 & Deo sancto Mercurio & $A E 1977,22^{76}$ & III sec., metà \\
\hline A66 & $\begin{array}{l}\text { Aug. } \\
\text { Minenae Aug. }\end{array}$ & 268 frt $\quad 3004^{77}$ & $57 \mathrm{dC}$ \\
\hline$A 67$ & Minervae Aug. & $401^{78}$ & 139 d.C. \\
\hline A68 & Neptuno Aug. & 536 cfr. 30786, pp. & II sec., metà \\
\hline A69 & [Pa]ntheo Aug. & 559 cfr. p. $3757^{80}$ & 161-169 d.C. \\
\hline A70 & Paci August. & $\begin{array}{l}199 \text { cfr. } 30712.36747, p p . \\
3004.3757^{81}\end{array}$ & 71 d.C.? \\
\hline A71 & Pietati Augustae & 562 cfr. pp. $3005 \cdot 3757^{82}$ & 22 d.C.; 43 d.C \\
\hline
\end{tabular}

${ }^{73}$ Ara o base (perduta) dal compitum di Via Marmorata: LTUR IV (Roma 1999) 260 s. (ivi altra bibliografia). La data del 3/2 a.C. (non dell' 1 a.C.) si ricava dall'anno V dell'era compitale e dai Fasti dei magistri.

${ }^{74}$ G. Mancini, Notizie degli Scavi di Antichità (1913), 117; G. Gatti, Bullettino della Commissione Archeologica Comunale di Roma 41 (1913), 77; E. Gatti, Studi Romani 1 (1913), 352 s., fig. 10. Vedi anche Hermann 1961, op. cit. (n. 45), 63; Schraudolph 1993, op.cit. (n. 37), 147 M 5, tav. 9. Datazione su base paleografica e stilistica.

${ }^{75}$ Frammento d'ara trovata a S. Cosimato di Trastevere. Perduta. Datazione puramente congetturale.

${ }^{76}$ S. Panciera, Rendiconti della Pontificia Accademia Romana di Archeologia 48 (1975/76), 302-308, cfr. W. Eck, Zeitschrift für Papyrologie und Epigraphik 37 (1980), 45-48. Base d'ignota provenienza. Datazione su base prosopografica.

${ }^{77}$ Foto e bibliografia in Supplementa Italica. Imagines 1999, op.cit. (n. 10), nr. 120. Ara di magistri fontani dall'Esquilino. Datazione consolare.

${ }_{78}^{7}$ Architrave (?) d'ignota provenienza che non so se ancora si conservi, almeno in parte, nel convento di S. Gregorio al Celio. Datazione consolare.

${ }^{79}$ ILS 3284. A. Arnaldi, Ricerche storico epigrafiche sul culto di Neptunus nell'Italia romana (Roma 1997), 93-98, nr. 3, fig. 2 a-c, vedi anche AE 1992, 114. Arula da fuori Porta Latina. La data è suggerita dal nome del dedicante che è un liberto di Antonino Pio.

${ }^{80}$ LS 1383. Ara di provenienza ignota, ora nei Musei Vaticani, Cortile della Pigna. La data si ricava dalla qualifica del dedicante come procurator Augustor(um) et Faustinae Aug. e dalle altre cariche ricoperte: H. Devijver, Prosopographia militiarum equestrium, I (Leuven 1976), 484 s. nr. 120; IV (Leuven 1987), $1615 \mathrm{nr} .120$; V (Leuven 1993), $2147 \mathrm{nr} .120$.

${ }^{81}$ Una datazione nel 71 è sostenuta dal confronto con le altre basi trovate insieme nel Foro Romano (CIL VI 196-198, 200), tra cui CIL VI 200 è ora nuovamente datata con diversi argomenti al 17 novembre di quest'anno da De Angeli e da G. Di Vita-Evrard, in un contributo in corso di stampa negli Atti del Colloquio Borghesi 2001, cit. (n. 4). Poiché d'altronde M. Arrecinus Clemens non poté dare il suo assenso all'erezione del monumento che come membro (in quanto pretore) della commissione augustea di sorveglianza sulle regioni, si dovrebbe ammettere ch'egli sia stato fatto prefetto prima della pretura e che abbia assunto quest'ultima nel 71 lasciando il pretorio a Tito: a favore Passerini, Athenaeum 18 (1940), 152 ss.. Contra G. Alföldy, Fasti Hispanienses (Wiesbaden 1969), 22; G. Mennella, Athenaeum 59 (1981), 205-208. A meno che la data di erezione non coincida con quella dell'autorizzazione (da notare che, contrariamente all'uso, nell'iscrizione si sarebbe letto solo permissu M. Arrecini Clementis senza indicazione della carica; forse perché questa non era più attuale al momento della messa in opera del monumento?). Per la registrazione negli Atti degli Arvali di sacrifici ad aram Pacis Augustae, vedi sopra in $\mathrm{n} .10$. 


\begin{tabular}{|l|l|l|l|}
\hline Nr. Id. & Divinità & Bibliografia & Datazione \\
\hline A72 & Saluti Aug. & 30983 cfr. p. $3758^{83}$ & età adrianea \\
A73 & Silvano Aug. & 634 cfr. $30804, \mathrm{pp} .3006 .3757^{84}$ & età traianea \\
A74 & Silvano Aug. & $635 \mathrm{cfr} .30805, \mathrm{pp} .835 .3006^{85}$ & 141 d.C. \\
A75 & Silvano Aug. & $36868^{86}$ & II sec., II metà \\
A76 & Silvano Aug. & $36825^{87}$ & II sec., II metà/III sec., \\
& & I metà \\
A77 & Sancto Silvano Aug. & 637 cfr. p. $3006^{88}$ & II/III sec., in. \\
A78 & Silvano Aug. & $636^{89}$ & $?$ \\
A79 & Silvano Aug. & 633 cfr. 30803, p. $3006^{80}$ & $?$ \\
A80 & Spei Aug. & 760 cfr. pp. $3006.3757^{91}$ & età traianea-adrianea \\
A81 & Spei Aug. & 759 cfr. $30826, p p .3006 .3757^{92}$ & $?$ \\
A82 & Statae Matri August. & $764^{93}$ & $6 / 5$ a.C. \\
A83 & Statae Matri Augustae & 802 cfr. pp. $3007.3757^{94}$ & 3/2 a.C. \\
\hline
\end{tabular}

${ }^{82}$ ILS 202. Un riepilogo dell'ampia discussione sul rapporto tra questa epigrafe vista dall'anonimo di Einsiedeln sul Campidoglio e la c.d. Ara Pietatis: LTUR IV (Roma 1999) 87-89, con tutta la principale bibliografia. Datazioni consolari.

${ }^{83}$ Vedi sopra A2 con n. 13 ed inoltre in n. 10 (Atti degli Arvali).

${ }^{84}$ ILS 1540a. Ara (?) d'ignota provenienza, ma forse da connettere con il vicus Frumentarius: LTUR V (Roma 1999), $166 \mathrm{~s}$; da qui viene altra dedica dello stesso schiavo di Traiano che ha posto la nostra (CL VI 544 cfr. p. 3005 , 3757; ILS 1540).

${ }^{85}$ A.E. Gordon, 1964, op. cit. (n. 31$) 75$ nr. 203, tav. 92 b-c. Base dal Quirinale: LTUR IV (Roma 1999), 316. Data consolare; il giorno della dedica (24 maggio) corrisponde al natale di Germanico, celebrato anche in età tarda: P. Herz 1975, op. cit. (n. 16), 327 cfr. 212.

${ }^{86}$ Vedi sopra A 12 con n. 23. Restauro di un signum di Silvano Augusto nel 185-192 d.C., vedi sopra in n. 10.

${ }^{87}$ Piccola base dal Celio nei pressi dei castra peregrinorum: LTUR IV (Roma 1999), 312. Il dedicante è un veter(anus) Augg.

${ }^{88}$ Rilievo di provenienza ignota riedito di recente, con diversa valutazione cronologica, da : R. Santolini Giordani, Antichità Casali (Roma 1989), 124 nr. 70, tav. XI (età severiana). Schraudolph 1993, op. cit. (n. 10), 169, S 1, tav. 14 (età tetrarchica).

${ }^{89}$ Ara o base in travertino di ignota provenienza. Perduta. Organizzazione collegiale. Dedicante C. Iulius Florentinus. Forse I/II sec. d.C.

${ }^{90}$ Ara o base d'ignota provenienza a Mannheim?. Dedicante un Aurelius senza prenome. Forse II/III sec. d.C.

${ }^{91}$.ILS 3772. Ara trovata verosimilmente presso l'arco di S. Lorenzo alla Marmorata: E. Rodriguez Almeida 1984, op.cit. (n. 70), 101 n. 3. Dalla foto del pezzo, che si conserva ancora nel Museo Despuig a Maiorca (ringrazio la collega Anna Pasqualini per avermene dato copia), non escluderei che la Regio indicata non sia la XII, ma coerentemente la XIII, con un'asta del numerale sulla comice. Non oltre l'età adrianea perché è ancora funzionante la commissione augustea di sorveglianza sulle regioni, vedi sopra A43 con n. 54. Il pretore T. Catius Catullinus Sestius Secundinus non è altrimenti conosciuto.

${ }^{92}$ Ara di provenienza ignota. Perduta? La dedica viciniae fa pensare ad un contesto compitale e, forse, ad una datazione alta.

${ }^{93}$ Piccola base certamente trovata alla fine del XVII sec. nel fondo Capobianco circa $400 \mathrm{~m}$. a NNO del km. 14 della via Nomentana (L. Quilici - St. Quilici Gigli, Ficulea (Roma 1993), 211 con n. 487) ma ricondotta a Roma per il contenuto che fa pensare a una dedica compitale. Non ne fu persuaso il Lanciani, seguito dai Quilici. L'iscrizione, che passò per il Museo Borgiano (vedi A. Russi, Epigraphica, 40[1978], 134 n. 20 ed ora F. Nasti, Iscrizioni latine del Museo di Napoli, I (Napoli 2000), 51 n. 49) non è perduta, ma si conserva all'Antiquarium Comunale del Celio, NCE 4265. Datazione desunta dall'anno $2^{\circ}$ dell'era vicana. 


\begin{tabular}{|l|l|l|l|}
\hline Nr. Id. & Divinità & Bibliografia & Datazione \\
\hline A84 & Statae Fortunae Aug. & 761 cfr. pp. $3006.3757^{95}$ & 12 d.C. \\
A85 & Statae Matri Aug. & 766 cfr. pp. $3006.3757^{96}$ & 44 d.C. \\
A86 & Veneri August. & AE $1980,54^{97}$ & I sec., fine \\
A87 & Victoriae Au[g.] & $791^{98}$ & 115 d.C. \\
A88 & Victoriae Augustae & $31403-31404$ cfr. pp. $3096 \mathrm{sg} .$. & $365-367$ d.C. \\
& & 4345 sg. & \\
A89 & Volcano Quieto Augusto & 802 cfr. pp. $3007.3757^{100}$ & $3 / 2$ a.C. \\
A90 & Volcano Quieto Augusto & 801 cfr. p. $3757^{101}$ & I sec., II metà \\
\hline
\end{tabular}

La prima considerazione che s'impone considerando questa lista è che $\mathrm{i}$ documenti non sono moltissimi, sia in rapporto alle circa 1800 iscrizioni sacre di Roma complessivamente disponibili ( 1 ventesimo), sia rispetto alle figure divine in qualche modo venerate a Roma, circa 200. Le divinità auguste cui sono rivolte le dediche non sono infatti più di 25 . La parte del leone è fatta dai Lares Augusti con ben 33 dediche, ${ }^{102}$ seguono Hercules e Silvanus con 6, Mercurius con 5, Fortuna e Diana con 4, Apollo e Stata Mater con 3, Aesculapius, Concordia, Mars, Spes, Victoria e Volcanus con 2; seguono altre 10 divinità con una sola attestazione ciascuna.

Sarebbe stato lecito aspettarsi - credo -, non dico una minor preminenza dei Lares Augusti che, vista l'importanza del loro culto in tutti i compita di Roma, doveva considerarsi scontata, quanto piuttosto la presenza di più

\footnotetext{
${ }^{94}$ ILS 3306. A Parma, Iscrizioni latine Napoli 2000, op.cit. (n.93), 68 nr. 18 con foto a p. 273. Lastra dal compitum del vicus Armilustri sull'Aventino: LTUR I (Roma 1993), 126 s. Per l'appartenenza al Museo Borgiano vedi $n$. prec. La data si ricava dall'anno $5^{\circ}$ dell'era compitale.

${ }^{95}$ ILS 3308. Ara o base del compitum dal vicus Sandalarius: LTUR V (Roma 1999), 189. Perduta. Datazione consolare.

${ }^{96}$ ILS 3309; Friggeri 2001, op. cit. (n. 18), 73 fig. b. Ara o base dal compitum del vicus Minervi: LTUR V (Roma 1999), $180 \mathrm{~s}$. La data è ricavata dall'anno 50 dell'era vicana (cfr. CIL VI 343: anno $32^{\circ}=26$ d.C.).

${ }^{97}$ S. Panciera, in Archeologia Laziale, 3 (1980), 203-206 con foto. Base dallo stesso compitum da cui viene A $65, \mathrm{n}$. 76. La datazione si ricava dal dedicante che fu magister vici una prima volta nel $7 / 6$ a.C. ed una seconda nel $4 / 5$ d.C. Qui appare come magister ter(tium), ma questa qualifica rappresenta un'aggiunta alla stesura originaria.

${ }^{98}$ ILS 1633; J. Vihonen, Iscrizioni latine Napoli, 2000, op. cit. (n. 93), 67 s. nr. 17 con foto a 273. Lastra (da base) scavata alla Moneta vedi sopra A5. Datazione consolare. Per l'offerta di una Victoria Augusta nella schola degli scribi librari e dei preconi, vedi sopra in nt. 10.

${ }^{99}$ G. Alföldy, Aurea Roma. Dalla città pagana alla città cristiana (Roma 2000), 463 con foto. Basi di statue dal ponte di Valentiniano; LTUR IV, (Roma 1999), $107 \mathrm{~s}$. Datazione dal nome (Simmaco) e dalla funzione del dedicante.

${ }^{100} \mathrm{ILS}$ 3306. Per provenienza, bibliografia e datazione, vedi sopra n. 94.

${ }^{101}$ ILS 3305. Epistilio perduto dal compitum del vicus Sabuci: LTUR V (Roma 1999), 185 s. Datazione ricavata dal numero, peraltro incompleto, almeno $50^{\circ}$, dell'era compitale.

${ }^{102}$ In realtà di più perché nell'elenco non sono state incluse le dediche compitali che non conservassero almeno parte del nome delle divinità.
} 
divinità o comunque di un maggior numero di attestazioni di culto per le divinità presenti. Si suole ritenere che, con il passare del tempo, l'epiteto Augustus / Augusta si sia banalizzato e pertanto sia stato generosamente dispensato senza guardare troppo per il sottile ad un gran numero di dei. Questo non è certamente il caso di Roma, che da questo punto di vista risulta, se mai, in forte controtendenza. Un esempio limite è fornito da Silvanus, di gran lunga la divinità più venerata a Roma in età imperiale (252 dediche secondo un mio recente censimento). ${ }^{103}$ Ebbene soltanto in 6 di queste 252 dediche, tutte di età imperiale, al dio viene attribuito l'epiteto di Augustus $(2,38 \%)$. Ad analoga conclusione si arriva considerando l'altra divinità, Hercules, che per sei volte appare con il titolo di Augustus: le dediche romane a questo dio sono infatti più di $150 \mathrm{ed}$, anche ammettendo con larghezza che un terzo siano di età repubblicana (sono certamente di meno), resterebbe che Ercole sarebbe detto Augustus in non più del $6 \%$ dei casi. Il confronto potrebbe essere esteso con analoghi risultati alle altre divinità del gruppo. Aggiungerei solo il caso di Mercurio perché un libro recente sul culto di Mercurio in Spagna, contenendo comparazioni, da un lato con le province gallico-germaniche, dall'altro con quelle africane, ${ }^{104}$ consente d'inserirlo agevolmente in un quadro più vasto. In totale le dediche di Mercurio a Roma sono 37, quelle a Mercurio Augusto sono 6. Data l'importanza del dio tra le divinità auguste, la percentuale sale in modo facilmente prevedibile al 16, 21 $\%$. Ma è interessante il confronto con gli altri ambiti sopra ricordati. Il totale delle dediche a Mercurio in Spagna è di 51; tra esse quelle a Mercurio Augusto (13) rappresentano il $25,49 \%$ quasi 10 punti più che a Roma). E la percentuale sale ulteriormente al $35,38 \%$ in Africa (con 130 documenti), mentre crolla al $4,34 \%$ tra Gallie e Germanie, province che pure hanno restituito ben 460 iscrizioni a Mercurio, peraltro in molti casi da considerare interpretatio romana di un dio locale.

Anche ammesso che questi dati (che non ho verificato) siano soggetti a qualche modifica, la disparità di comportamento da luogo a luogo non credo sia in discussione. Ne consegue che, non solo l'epiteto non è così inflazionato come si ritiene, ma la sua stessa presenza / assenza merita attenzione ${ }^{105}$ come pure la ragione che di volta in volta può averla determinata.

\footnotetext{
103 'Silvano a Roma', in Studia in honorem Georgii Mihailov (Sofia 1995 [1996]), 347-361 (248 testi); adde $\mathrm{AE} 1990,56$ e 118; 1991, 295; 1993, 152.

${ }^{104} \mathrm{G}$. Baratta, Il culto di Mercurio nella penisola iberica (Barcelona 2001), 108, con n. 356.

${ }^{105}$ Degno di nota, ad esempio, che l'epiteto Augustus/ $-a$ non è attribuito non solo ad alcuna delle divinità menzionate negli Atti dei Ludi secolari del 17 a.C. (Apollo, Diana, Hercules Victor, Ilithya, Iuno, Iuno Regina, Iuppiter Optimus Maximus, Iuppiter Stator, Iuppiter Tonans, Latona, Moerae, Ops, Terra Mater)
} 
Per quanto riguarda le divinità auguste nel loro complesso, il confronto con il libro di Étienne mostra, con alcune coincidenze, anche cospicue differenze. Dico le liste, non la lista, di Étienne perché, mentre qui per comodità si è preferito redigerne una sola, egli ha scelto di compilarne due: una di quelle che, seguendo il Mattingly, il Charlesworth e il Nock, definisce delle virtù imperiali ${ }^{106}$ e l'altra delle divinità auguste vere e proprie. Non senza motivo, perché si tratta di figure religiose diverse anche se non sempre un preciso confine risulta facilmente tracciabile. Il Fishwick propone a sua volta di dividere in due la lista delle cosiddette virtù, mettendo da una parte quelle che considera le virtù imperiali vere, come l'Aequitas, la Clementia, la Constantia, la Fides, l'Indulgentia, la Iustitia e così via e dall'altra quelli che possono considerarsi piuttosto i risultati, i benefici dell'azione imperiale come la Libertas, la Pax, la Salus, la Spes e quant'altro. ${ }^{107}$ Anche questa divisione non è comunque esente da qualche problema.

Da un confronto della lista Étienne delle virtù con la nostra risulta che sono presenti in comune Concordia, Fortuna, Pax, Pietas, Salus, Victoria. Assai diverso è però in vari casi il numero delle attestazioni: 2 a Roma contro 5 in Spagna per Concordia; 1 contro 5 per Pietas; 1 contro 7 per Salus; 2 contro 11 per Victoria. Mancano inoltre nella lista romana Aeternitas, Bonus Eventus, Iuventus, Libertas, Providentia, ${ }^{108}$ Tutela, Virtus; mentre in quella spagnola non c'è Spes, peraltro presente a Roma solo 2 volte. Da notare che in entrambe le liste mancano virtù "canoniche" imperiali (augustee in particolare) come Clementia e Iustitia. ${ }^{109}$ Pur facendo qualche parte all'azzardo dei ritrovamenti, non si può fare a meno di constatare che il fenomeno romano risulta assai più ridotto, sia quantitativamente, sia

sui quali vedi ora B. Schnegg - Köhler, Die augusteischen Säkularspiele. Archiv für Religionsgeschichte 4 (München-Leipzig 2002), ma neppure ad alcuna delle molte divinità pregate per Augusto dal liberto $L$. Lucretius Zethus nell'1 d.C.: CIL VI 30975 = ILS 3090; cfr. M. A. Cavallaro, Helikon 15-16 (19751976), 146-186. Nessuna divinità augusta neppure nei Ludi Seculari severiani: G. B. Pighi, De ludibus saecularibus populi Romani Quiritium (2a ed., Amsterdam 1965).

106 H. Mattingly, 'The Roman Virtues', Harvard Theological Review 30 (1937), 103-117; M.P. Charlesworth, 'The Virtues of a Roman Emperor', Proceedings of the British Academy 23 (1937), 105133; A. D. Nock, 'The Emperor's Divine Comes', Joumal of Roman Studies 37 (1947), 112-116 (Idem, Essays on Religion and the Ancient World [Oxford 1972], 670-675).

${ }^{107}$ Fishwick 1991, op. cit. (n. 1), 454-474, in part. 460. Alla bibliografia sulle "virtu" imperiali indicata nella nota precedente ed a quella registrata in Fishwick, aggiungere: C. V. Classen, "Virtutes imperatoriae', Arctos 25 (1991), 17-39; C. F. Noreña, 'The Communication of Emperor's Virtues', Joumal of Roman Studies 91 (2001), 146-168.

${ }^{108}$ Per la quale vedi però sopra in $\mathrm{n} .10$.

${ }^{109}$ Contro l'idea di un canone di virtù imperiali di origine filosofica formato da virtus, clementia, iustitia e pietas vedi in particolare A. Wallace- Hadrill, 'The Emperor and his Virtues', Historia 30 (1981), 298323 e Classen 1991, art. cit. (n. 107). 
qualitativamente di quello spagnolo, come se la disponibilità a questo tipo di culto, mutato l'ambiente, fosse più ridotta e comunque diversamente orientata.

Nella stessa direzione si è condotti da un confronto della lista Étienne delle divinità auguste con la nostra. Ancora una volta troviamo divinità presenti in entrambe: Aesculapius, Apollo, Diana, Hercules, Isis, Mars, Mercurius, Minerva, Neptunus, Pantheus, Silvanus, Venus. Ma in certi casi il numero delle testimonianze è marcatamente diverso: Hercules e Silvanus (6 a Roma e 3 in Spagna); però Mars (2 a Roma 16 in Spagna). Mancano d'altronde nella lista romana Caelestis, Iuno, Iuppiter (presente solo il Maleciabrudes e l'Heliopolitamus), Liber Pater, Luna, Lupa, Nemesis, Pollux, Sol e Vesta, mentre nella lista spagnola sono assenti Fons, Iuppiter Maleciabrudes, Iuppiter Heliopolitanus, Volcanus ed i Lares che invece hanno 33 testimonianze a Roma, dando l'impressione di aver concentrato su di sé un culto altrove più diffuso o caratterizzato da altri addensamenti (Mars, Victoria, Venus, Salus, ad esempio).

Consideriamo ora la seconda tabella, in cui le dediche alle divinità auguste sono ordinate cronologicamente.

\section{B - Secondo cronologia}

\begin{tabular}{|c|c|c|}
\hline Nr. Id. & Datazione & Divinità \\
\hline$B 1=A 8$ & $7 / 6$ a.C. & [D]ianae August. \\
\hline$B 2=A 26$ & 7/6 a.C. & Lanibus Augustis \\
\hline$B 3=A 27$ & 7/6 a.c. & Larib. Aug. \\
\hline$B 4=A 28$ & $7 / 6$ a.C. & Larib. Aug. \\
\hline$B 5=A 29$ & 7/6 a.c. & Larib[us Aug.] \\
\hline$B 6=A 61$ & 7/6 a.C. & Mercurio Augus[to] \\
\hline$B 7=A 82$ & $6 / 5$ a.C. & Statae Matri August. \\
\hline$B 8=A 62$ & $3 / 2$ a.C. & Mercurio Aug. \\
\hline$B 9=A 83$ & $3 / 2$ a.C. & Statae Matri Augustae \\
\hline$B 10=A 89$ & $3 / 2$ a.C. & Volcano Quieto Augusto \\
\hline$B 11=A 30$ & 2 a.c. & Laribus Augustis \\
\hline$B 12=A 31$ & 2 a.c. & Laribus Augustis \\
\hline$B 13=A 3$ & $2 / 1$ a.C. & Apollini Aug. \\
\hline$B 14=A 9$ & 1 a.C./1 d.C. & Dianae Augustae \\
\hline$B 15=A 86$ & I sec. a.C., fine & Veneri August. \\
\hline$B 16=A 32$ & $2 / 3$ d.C. & Laribus Augustis \\
\hline$B 17=A 33$ & $3 / 4$ d.C. & Lar. Aug. \\
\hline$B 18=A 84$ & 12 d.C. & Statae Fortunae Aug. \\
\hline$B 19=A 6$ & età augustea & [Conc]ord[iae] Aug. \\
\hline$B 20=A 10$ & età augustea & Dianae Augustae \\
\hline$B 21=A 34$ & età augustea & Lar. [Aug.] \\
\hline$B 22=A 63$ & età augustea & Mercurio Aug. \\
\hline $\mathrm{B} 23=\mathrm{A} 1$ & 25 d.C. & Aisculapio Augusto \\
\hline$B 24=A 17$ & 26 d.C. & [He]rculi Tuta[tori A]ug. \\
\hline
\end{tabular}




\begin{tabular}{|c|c|c|}
\hline Nr. Id. & Datazione & Divinità \\
\hline$B 25=A 71$ & 22 d.C.; 43 d.C. & Pietati Augustae \\
\hline$B 26=A 85$ & 44 d.C. & Statae Matri Aug. \\
\hline$B 27=A 4$ & 46 d.C. & Apollini Aug. \\
\hline$B 28=A 7$ & I sec., I metà & [Co]ncordiae Aug. \\
\hline $\mathrm{B} 29=\mathrm{A} 36$ & I sec., I metà & Laribu[s Aug.] \\
\hline$B 30=A 59$ & I sec., I metà & Mar[ti] Aug. \\
\hline$B 31=A 35$ & età tiberio - claudiana & Laribus Augusti[s] \\
\hline$B 32=A 37$ & 56 d.C.? & Laribus Augustis \\
\hline$B 33=A 66$ & 57 d.C. & Minervae Aug. \\
\hline$B 34=A 70$ & 71 d.C.? & Paci August. \\
\hline $\mathrm{B} 35=\mathrm{A} 18$ & età vespasianea & Herculi Aug. \\
\hline$B 36=A 38$ & 83 d.C. & Laribus Aug. \\
\hline $\mathrm{B} 37=\mathrm{A} 39$ & età domizianea & Laribu[s Aug.] \\
\hline $\mathrm{B} 38=\mathrm{A} 40$ & 98/99 d.C. & Laribus Augustis \\
\hline $\mathrm{B} 39=\mathrm{A} 41$ & I sec. d.C. & La[ribus] A[ugustis] \\
\hline$B 40=A 64$ & I sec. d.C. & Merc. Aug. \\
\hline$B 41=A 42$ & | sec., || metà & Laribus Aug. \\
\hline $\mathrm{B} 42=\mathrm{A} 90$ & | sec., II metà & Volcano Quieto Augusto \\
\hline $\mathrm{B} 43=\mathrm{A} 43$ & I sec., fine/ll inizio & [Laribu]s Aug. \\
\hline$B 44=A 44$ & 100 d.C. & Laribus Augustis \\
\hline $\mathrm{B} 45=\mathrm{A} 45$ & 109 d.C. & [Laribus A]ugustis \\
\hline$B 46=A 5$ & 115 d.C. & Apollini Aug. \\
\hline $\mathrm{B} 47=\mathrm{A} 14$ & 115 d.C. & Fortunae Aug. \\
\hline$B 48=A 21$ & 115 d.C. & Herculi Aug. \\
\hline$B 49=A 87$ & 115 d.C. & Victoriae Au[g.] \\
\hline$B 50=A 46$ & 116 d.C. & La[rum Aug.] \\
\hline $\mathrm{B} 51=\mathrm{A} 22$ & età traianea & Herculi Aug. \\
\hline$B 52=A 73$ & età traianea & Silvano Aug. \\
\hline$B 53=A 2$ & età adrianea & Aesculapio Aug. \\
\hline $\mathrm{B} 54=\mathrm{A} 72$ & età adrianea & Saluti Aug. \\
\hline$B 55=A 80$ & età traianea-adrianea & Spei Aug. \\
\hline$B 56=A 67$ & 139 d.C. & Minervae Aug. \\
\hline$B 57=A 74$ & 141 d.C. & Silvano Aug. \\
\hline$B 58=A 47$ & 149 d.C. & [Laribus Aug]ustis \\
\hline$B 59=A 48$ & 161 d.C. & [Laribus Augusto]rum \\
\hline$B 60=A 49$ & 169 d.C. & [Larib]us Augg. \\
\hline$B 61=A 69$ & 161 - 169 d.C. & [Pa]ntheo Aug. \\
\hline$B 62=A 22$ & età commodiana & Herculi August. \\
\hline$B 63=A 13$ & I/II sec. d.C. & Fort[unae] Aug. \\
\hline$B 64=A 20$ & |l/l sec. d.C. & Herculi Aug. \\
\hline$B 65=A 60$ & |l/l sec. d.C. & Marti Aug. \\
\hline$B 66=A 23$ & II sec. & [Isi]di Aug. \\
\hline$B 67=A 50$ & II sec. & Laribus Aug. \\
\hline$B 68=A 68$ & II sec., metà & Neptuno Aug. \\
\hline$B 69=A 11$ & II sec., II metà & [Di]anae Aug. \\
\hline$B 70=A 75$ & II sec., II metà & Silvano Aug. \\
\hline $\mathrm{B} 71=\mathrm{A} 25$ & II sec., fine & I.O.M.H. Aug. \\
\hline $\mathrm{B} 72=\mathrm{A} 77$ & II sec., fine/III inizio & Sancto Silvano Aug. \\
\hline$B 73=A 51$ & 203 d.C. & Larib. Aug. \\
\hline $\mathrm{B} 74=\mathrm{A} 52$ & 223 d.C. & Laribus Aug. \\
\hline
\end{tabular}




\begin{tabular}{|l|l|l|}
\hline Nr. Id. & Datazione & Divinità \\
\hline B75 = A53 & $222-235$ d.C. & [La]ribus Alugustis] \\
B76 = A12 & $244-249$ d.C. & Fonti Aug. \\
B77 = A76 & II sec., II metà / III sec., I metà & Silvano Aug. \\
B78 = A15 & II sec. fine / III sec. & Fortunae Augustae Respicie[nti] \\
B79 = A16 & II sec. fine / III sec. & Fortunae Augustae Praesenti \\
B80 = A65 & III sec., metà & Deo Sancto Mercurio Aug. \\
B81 = A24 & III sec. & lovi Maleciabrudi Aug. \\
B82 = A88 & $365-367$ d.C. & Victoriae Augustae \\
B83 = A54 & $?$ & Laribus Aug. \\
B84 = A55 & $?$ & Larib. Aug. \\
B85 = A56 & $?$ & Laribus Augustis \\
B86 = A57 & $?$ & Laribus Aug. \\
B87 = A58 & $?$ & [La]ribus Augustis \\
B88 = A78 & $?$ & Silvano Aug. \\
B89 = A79 & $?$ & Silvano Aug. \\
B90 = A81 & $?$ & Spei Aug.. \\
\hline
\end{tabular}

In base a questa lista si dovrebbe affermare che l'arco temporale in cui le divinità auguste sono documentate è assai ampio: dall'ultimo decennio a.C. ad almeno il sesto decennio del IV sec. d. C. In realtà dediche alla Victoria Augusta dal ponte di Valentiniano a parte, ${ }^{110}$ documenti che per più versi costituiscono un caso a sé (tra l'altro doveva esser stata rimessa da poco nella curia l'ara della Vittoria che Costanzo II aveva fatto togliere nel $357^{111}$ ), non si hanno sostanzialmente più dediche a divinità auguste dopo il III sec. o addirittura dopo la metà di quel secolo. L'ultima delle dediche ai Lares Augusti che, con il loro numero, costituiscono uno straordinario esempio di continuità, è databile agli anni 222-235. In Spagna l'ultimo esempio datato è, secondo Étienne, del tempo di Caracalla ${ }^{112}$ o meglio di età antoniniana, ${ }^{113}$ ivi stesso le dediche a Mercurio Augusto, secondo le ricerche della Baratta sono per lo più dei secoli I e II con qualche incerta espansione nel III limitatamente alla Gallaecia ed alla Lusitania. ${ }^{114}$ L'ultima dedica datata a Mercurio Augusto in Germania è del 249. ${ }^{115}$

\footnotetext{
${ }^{110}$ Vedi sopra con $n .99$.

${ }^{111}$ Sulla rimozione dell'ara (e della statua) per volontà di Costanzo II ed il suo ripristino al tempo, come si ritiene, di Giuliano con le fonti pertinenti: S. Mazzarino, 'Tolleranza e intolleranza: la polemica dell'ara della Vittoria', Antico, tardoantico ed èra costantiniana I (Bari 1974), 339-377, in part. 339-357 (p. 352 n. 32 per il ripristino); D. Vera, Commento storico alle Relationes di Quinto Aurelio Simmaco (Pisa $1981), 12 \mathrm{~s}$.

${ }^{112}$ CIL II 2121; Étienne 1958, op. cit. (n. 8), 340 n. 9.

${ }^{113} \mathrm{CLL} \mathrm{\Pi ^{2 }} / 7,56$.

${ }^{114}$ Baratta 2001, op. cit. (n. 104), 108

${ }^{115}$ CIL XIII 6658; Liertz 1998, op. cit. (n.9), 174, 176.
} 
Va considerato inoltre che, tra I e III sec., allo stato almeno della nostra documentazione, le divinità auguste sono lontane dal presentare a Roma uno scaglionamento uniforme. L'unica attestazione di Venus è della fine del I sec. a.C.; Stata Mater, Concordia, Pietas, non oltrepassano la metà del I sec. d.C.; Volcanus e Pax non superano la fine del I sec.; Aesculapius, Apollo, Neptunus, Salus, non vanno oltre la metà del II: Diana, Hercules, Mars, Pantheus, non oltrepassano il II; Fortuna, Mercurius e Fons (come i Lares), non superano forse la metà circa del III sec. Altre divinità invece presentano l'epiteto solo tardi come Silvamus per cui non è attestato prima dell'età traianea, e Iuppiter Maleciabrudes ed Heliopolitamus, per cui l'abbiamo solo dalla fine del II o inizio del III sec. Insomma anche dal punto di vista cronologico il paesaggio è meno piatto ed uniforme di quel che si potrebbe credere.

E veniamo ai dedicanti, analizzati nelle tabelle $\mathrm{C}$ e $\mathrm{D}$. Se, come sopra si è sospettato, l'attribuzione dell'epiteto Augustus / Augusta ad una determinata divinità non è irrilevante, si capisce l'importanza di registrare accuratamente da parte di chi l'attributo è conferito.

\section{C - Secondo dedicanti}

\begin{tabular}{|l|l|l|}
\hline $\mathrm{Nr}$. id. & tipo di dedicante & Dedica \\
\hline $\mathrm{C} 1$ & uomo/donna & tutti uomini \\
$\mathrm{C} 2$ & ingenuo & $\mathrm{B} 2, \mathrm{~B} 14, \mathrm{~B} 24, \mathrm{~B} 25, \mathrm{~B} 26, \mathrm{~B} 34, \mathrm{~B} 44, \mathrm{~B} 56, \mathrm{~B} 58, \mathrm{~B} 59$, \\
& & $\mathrm{B} 61, \mathrm{~B} 65, \mathrm{~B} 73, \mathrm{~B} 77, \mathrm{~B} 80, \mathrm{~B} 82$ \\
$\mathrm{C3}$ & servo/liberto imperiale & $\mathrm{B} 35, \mathrm{~B} 46, \mathrm{~B} 47, \mathrm{~B} 48,[\mathrm{~B} 51], \mathrm{B} 52, \mathrm{~B} 60, \mathrm{~B} 64, \mathrm{~B} 68, \mathrm{~B} 76$ \\
$\mathrm{C} 4$ & servo/liberto non imperiale & $\mathrm{B} 3, \mathrm{~B} 4, \mathrm{~B} 5, \mathrm{~B} 6, \mathrm{~B} 8, \mathrm{~B} 11, \mathrm{~B} 12, \mathrm{~B} 13, \mathrm{~B} 14, \mathrm{~B} 15, \mathrm{~B} 16$, \\
& & $\mathrm{B} 17, \mathrm{~B} 18, \mathrm{~B} 23, \mathrm{~B} 27, \mathrm{~B} 34, \mathrm{~B} 36, \mathrm{~B} 38, \mathrm{~B} 40, \mathrm{~B} 42, \mathrm{~B} 44$, \\
$\mathrm{C} 5$ & inc. con cognome greco & $\mathrm{B} 45, \mathrm{~B} 48, \mathrm{~B} 53, \mathrm{~B} 58, \mathrm{~B} 59, \mathrm{~B} 70$ \\
& & $\mathrm{~B} 1, \mathrm{~B} 2, \mathrm{~B} 6, \mathrm{~B} 9, \mathrm{~B} 10, \mathrm{~B} 14, \mathrm{~B} 24, \mathrm{~B} 26, \mathrm{~B} 30, \mathrm{~B} 32, \mathrm{~B} 33$, \\
$\mathrm{C} 6$ & inc. con cognome latino & $\mathrm{B} 1, \mathrm{~B} 43, \mathrm{~B} 45, \mathrm{~B} 48, \mathrm{~B} 53, \mathrm{~B} 67, \mathrm{~B} 71, \mathrm{~B} 75, \mathrm{~B} 78, \mathrm{~B} 79$, \\
& & $\mathrm{B} 57, \mathrm{~B} 14, \mathrm{~B} 16, \mathrm{~B} 20, \mathrm{~B} 26, \mathrm{~B} 28, \mathrm{~B} 32, \mathrm{~B} 33, \mathrm{~B} 34, \mathrm{~B} 53$, \\
$\mathrm{C} 7$ & inc. senza cognome & $\mathrm{B} 2, \mathrm{~B} 29, \mathrm{~B} 42, \mathrm{~B} 49, \mathrm{~B} 69, \mathrm{~B} 70, \mathrm{~B} 75, \mathrm{~B} 76$ \\
\hline
\end{tabular}

Nella tabella C i dedicanti sono stati ripartiti secondo l'appartenenza ad alcuni gruppi oppositivi: uomo/donna o ingenuo/schiavo-liberto, distinguendo ulteriormente tra schiavi e liberti imperiali o di altri e mantenendo separati gli incerti, che tali sono in realtà, come vedremo, solo in esigua minoranza. ${ }^{116}$

Prima constatazione su questa lista: nessuna donna tra i dedicanti (C1). Che le donne costituiscano in epigrafia un gruppo di dedicanti fortemente

\footnotetext{
${ }^{116}$ La lista rende conto del numero dei documenti in cui i vari tipi di dedicanti occorrono e non di quello dei dedicanti, del resto non sempre accertabile.
} 
minoritario è fatto ben noto. Se consideriamo i dedicanti singoli di CIL VI, vediamo che in 613 casi sono uomini e solo in 50 donne (anche insieme appaiono poco: solo 62 volte). Qui va considerato però che non c'è scarsità, bensì totale assenza, come se venerare una divinità augusta fosse una pratica esclusivamente da uomini.

Seconda osservazione: ingenui certi (C2) appaiono soltanto in 16 dediche su 90. Inoltre solo in 4 casi, tutti per qualche verso anomali, ${ }^{117}$ il dedicante ingenuo appartiene ad uno strato sociale elevato o addirittura elevatissimo, come nella dedica pubblica B25 che è quella famosa alla Pietas Augusta eseguita nel 43 da Claudio secondo un senatoconsulto del 22 (non posso soffermarmi su questa intricata questione sulla quale ha fatto il punto da ultimo Eugenio La Rocca) ${ }^{118}$ o nel caso di B73 (dedica ai Lares Augusti di Settimio Severo e Caracalla). B61 è una dedica a Pantheo Augusto da parte di un procurator equestre di Marco Aurelio, Lucio Vero e di Faustina Augusta. La dedica B80 è fatta ex oraculo a Mercurio Augusto da un senatore di origine microasiatica (forse efesino) insieme con i suoi familiari, intorno alla metà del III sec. Da ultimo vengono le già ricordate dediche alla Vittoria Augusta poste sul ponte di Valentiniano tra il 10 marzo 365 e il 24 agosto 367 dall'ex prefetto urbano L. Aurelio Avianio Simmaco. In altri due casi il dedicante, verosimilmente ingenuo, è un veteramus: rispettivamente B65 a Marte e B77 a Silvano. Nei rimanenti 9 documenti l'ingenuo compare insieme con dedicante di condizione libertina, in particolare ( 7 casi) nell'ambito del culto compitale tra i cui magistri, per lo più liberti, potevano esserci eccezionalmente anche persone di nascita libera.

Tutti gli altri dedicanti sono schiavi o liberti, sicuri o molto probabili, ad esempio per il cognome greco o per il contesto (ad esempio quello del culto compitale in cui figurano).

Non sembra dunque inappropriato ricavare la conclusione ulteriore che a Roma il culto delle divinità auguste sia in misura nettamente preponderante un culto privato servile o libertino.

Con la tabella $\mathrm{D}$ possiamo anche verificare in quale veste $\mathrm{i}$ dedicanti dichiarino di comporre le loro offerte.

\footnotetext{
${ }^{117}$ Sono indicati sotto nella tabella $\mathrm{D}$ al $\mathrm{nr} .21$ (=B25, 61, 80, 82).

${ }^{118}$ LTUR IV (Roma 1999), 87-89.
} 


\begin{tabular}{|c|c|c|}
\hline Nr. id. & qualifica dedicante & Dedica \\
\hline D1 & collegium struc[torum] & B85 \\
\hline $\mathrm{D} 2$ & $\begin{array}{l}\text { collegium Larum praedio[rum - et] Dianae } \\
\text { Bat[-]e... [per - - - cur]atorem et Eutychum } \\
\text { adiuto[rem] }\end{array}$ & B60 \\
\hline D3 & $\begin{array}{l}\text { conduct[ores] flaturae argen[tar(iae)] monetae } \\
\text { Cae[saris] }\end{array}$ & B49 \\
\hline D4 & curatores tribus Suc(cusanae) lunior(um) & B34 \\
\hline D5 & disp(ensator) fisci fr(umentarii) & B52 \\
\hline D6 & magistri fontani & B33 \\
\hline D7 & magistri / ministri vici & $\begin{array}{l}\mathrm{B} 1, \mathrm{~B} 2, \mathrm{~B} 3, \mathrm{~B} 4, \mathrm{~B} 5, \mathrm{~B} 6, \mathrm{~B} 7, \mathrm{~B} 8, \mathrm{~B} 9, \\
\mathrm{~B} 10, \mathrm{~B} 11, \mathrm{~B} 12, \mathrm{~B} 13, \mathrm{~B} 14, \mathrm{~B} 16, \mathrm{~B} 17, \\
\mathrm{~B} 18, \mathrm{~B} 23, \mathrm{~B} 24, \mathrm{~B} 26, \mathrm{~B} 27, \mathrm{~B} 29, \mathrm{~B} 32, \\
\mathrm{~B} 36, \mathrm{~B} 38, \mathrm{~B} 42, \mathrm{~B} 43, \mathrm{~B} 44,[\mathrm{~B} 50], \mathrm{B} 58, \\
\mathrm{~B} 59, \mathrm{~B} 67,[\mathrm{~B} 73], \mathrm{B} 75, \mathrm{~B} 87\end{array}$ \\
\hline D8 & me(n)sor aedificiorum Augg. & $B 69, B 70$ \\
\hline D9 & $\begin{array}{l}\text { officinatores et nummularii officinarum } \\
\text { argentariarum familiae monetari(ae) }\end{array}$ & B51 \\
\hline D10 & $\begin{array}{l}\text { officinatores monetae aurariae argentariae } \\
\text { Caesaris n(ostri) }\end{array}$ & B47 \\
\hline D11 & optio et exactor auri argenti et aeris & B46, B47 \\
\hline D12 & $\begin{array}{l}\text { optio et exactor auri arg(enti) aeris item } \\
\text { signatores, suppostores, malleatores monetae } \\
\text { Caesaris n(ostri) }\end{array}$ & B48 \\
\hline D13 & pigmentarii et miniarii (?) & B28 \\
\hline D14 & pr(ocurator), lib. imp. & B64, B68 \\
\hline D15 & quaestor di comunità isiaca & B66 \\
\hline D16 & $\begin{array}{l}\text { quinquennalis perpetuus collegii iuvenum } \\
\text { Racilianensium }\end{array}$ & B62 \\
\hline D17 & sacerdos I.O.M.H. & $B 71$ \\
\hline D18 & tabul(arius) a marmoribus & B35 \\
\hline D19 & veter(anus), veter(anus) Augg. & B65, B77 \\
\hline $\mathrm{D} 20$ & $\begin{array}{l}\text { vilici praediorum Galbanorum et plebs, } \\
\text { collegium salutare }\end{array}$ & B53, B54 \\
\hline $\mathrm{D} 21$ & personalità eminenti & $\begin{array}{l}\text { B25 (imp. Claudio), B61 (procur. } \\
\text { equestre), B73 (Impp. Settimio } \\
\text { Severo e Caracalla), B80 (vir } \\
\text { clarissimus), B82 (ex praef. urb.) }\end{array}$ \\
\hline
\end{tabular}

$\mathrm{Da}$ questa lista risulta evidente che la maggior parte dei dedicanti sottolinea la sua appartenenza a gruppi che, o sono direttamente relazionabili alla figura imperiale, o desiderano essere messi in rapporto con essa. Oltre $\mathrm{i}$ numerosi magistri e ministri vici, cui competeva il culto dei Lares Augusti e del Genius Caesarum (D7) ed ai membri di un collegium Larum in una proprietà imperiale (D2) si segnalano tutto il personale della Moneta Caesaris 
(D3, D9, D10, D1 1, D12), ed altri membri a vario livello dell'amministrazione imperiale: un dispensator fisci frumentarii (D5), un mensor aedificiorum (D8), due procuratores libertini (D14), un tabularius a marmoribus (D18). Un collegium structorum, non meglio identificato (D1), fa una dedica dei Lares Augusti alle pendici del Palatino: sarà verosimilmente costituito da structores della casa imperiale. Una dedica alla Concordia Augusta ricorda che un sigmum della dea, cum suis ornamentis è stato offerto, nel contesto di un collegio di pigmentarii e miniarii insieme con imagines argenteas ((tres)), verosimilmente dell'imperatore e dei membri della sua famiglia (D13). Una dedica ad Ercole è posta da un quinquennalis perpetuus collegi iuvenum Racilianensium (D16); se coglie nel vero la mia vecchia interpretazione che mette in relazione questa organizzazione romana della iuventus con la suddivisione della città in regiones e vici (e in effetti esistono in vicus Raciliani maioris ed un vicus Raciliani minoris) ${ }^{119}$ siamo indirettamente ricondotti alle strutture (regiones, vici) che a Roma sono alla base del cosiddetto culto imperiale. Una dedica Aesculapio et Saluti Aug(ustae) o Aug(ustis) e fatta da un collegium salutare istituito tra il personale dei praedia Galbana (D20); primo destinatario della dedica è il numen della domus Augusta ed essa è posta loco adsignato dal procurator patrimoni Caesaris nostri. Lascio da parte qualche altro caso (D4, D6, D17, D19). Non mi sembra comunque dubbio che il culto delle divinità auguste trovi la sua diffusione a Roma, non solo quasi esclusivamente tra schiavi e liberti, ma anche tra schiavi e liberti strettamente collegati con la casa imperiale. Solo in parte analogo il quadro che Robert Étienne ricava dalla documentazione spagnola ${ }^{120}$ nella quale su 74 dedicanti solo 34 , cioè il $43,24 \%$, sono costituiti da schiavi (6), liberti (8), indigeni (1), seviri (15), magistri Larum (4).

Dopo questa rassegna, si può tornare a chiedersi, prima di concludere, che cosa significhino effettivamente queste dediche romane rispetto al problema generale che si è posto all'inizio: sono manifestazioni di culto per un imperatore-dio?; di culto congiunto per un dio e per l'imperatore?; di culto per un dio in veste di guida e patrono dell'imperatore?; o, infine, soltanto il prodotto di una mescolanza di devozione verso gli dei e di piaggeria nei confronti dell'uomo più potente tra i potenti?

Nessun segno mi sembra ravvisabile di vero e proprio culto imperiale, né in proprio, né sotto le sembianze delle varie divinità evocate. Escluderei che una dedica Mercurio Augusto possa essere equivalente ad una dedica Mercurio et

\footnotetext{
${ }^{119}$ Vedi sopra, n. 33.

${ }^{120}$ Étienne 1958, op. cit. (n. 8), 345.
} 
Augusto. ${ }^{121}$ È vero che si possono trovare nomi di divinità giustapposti in asindeto, ma in genere si tratta, per l'appunto, di più divinità (almeno tre) e non di due, tra le quali normalmente la congiunzione non manca, oltretutto per l'equivoco che (in casi come questo) altrimenti ne conseguiva.

Che quando si pone una dedica ad una divinità con l'epiteto di Augusta lo si faccia per pura piaggeria, al di fuori di ogni sentimento religioso, è d'altronde una di quelle affermazioni che, essendo indimostrabili, risultano storicamente inutilizzabili.

È certo invece che non possono riguardare, neppure indirettamente, un imperatore-dio vivente, né le dediche a divinità auguste poste dal sovrano stesso come quella della Pietas Augusta da parte di Claudio (A71), o quella ai Lares Augusti da parte di Settimio Severo e Caracalla (A51), né quelle fatte da altri a qualche divinità augusta pro salute dell'imperatore, tenuto ben distinto dal dio invocato, come nel caso di Fons per i Filippi (A12).

Le divinità sono d'altronde Augustae o Augusti? Ed è avvertibile nelle dediche romane un'autentica differenza di significato tra le due denominazioni?

Se si scorrono le liste che ho prodotto si vede bene che in tutto l'arco della documentazione, quando l'epiteto è scritto per esteso, esso si presenta nella quasi totalità dei casi in forma aggettivale. ${ }^{122}$ Non vedo motivo perché le abbreviazioni dovrebbero essere sciolte diversamente.

In un solo caso (B59) abbiamo sicuramente Laribus Augustorum (per esteso) invece che Augustis. In un altro (B60), abbiamo Augg. scritto con due $G$. Il motivo di queste eccezioni è facilmente riconoscibile: con le prime due dediche siamo negli anni della prima correggenza imperiale, quella di Marco Aurelio e Lucio Vero, con la terza, ipotetica, in quelli della correggenza di Settimio Severo e Caracalla. Credo che il ricorso alla forma genetivale invece che a quella aggettivale, da un lato sia imputabile alle novità della correggenza, dall'altro mostri che, se con due imperatori si ritiene opportuno scrivere Augustorum, in tutti gli altri casi in cui, con riferimento ad una divinità, si scrive Augustus / Augusta, l'epiteto non fosse sentito sostanzialmente diverso da Augusti, o anche, più tardi, da Augustorum. Del resto che, tanto con Augustus quanto con Augusti, non s'intenda istituire un rapporto con l'imperatore come istituzione, bensì concretamente con

\footnotetext{
${ }^{121}$ Cosi da ultimo Clauss 1999, op. cit. (n. 2), 280 e 286 ss. Contro questa interpretazione e, in generale, contro la tesi propugnata in questo libro di un vero e proprio culto generalizzato dell'imperatore vivente sin dal tempo di Augusto: C. Letta, Athenaeum 99 (2002), 625-632.

${ }^{122} \mathrm{~B} 2,9,10,11,12,14,16,20,23,25,32,38,42,44,45,58,78,79,82,85,87$. Non sono elencati i casi in cui la forma estesa è frutto di integrazione.
} 
l'imperatore regnante, ${ }^{123}$ è mostrato da dediche come la $\mathrm{A} 40$, la cui intitolazione, a scanso di equivoci, suona Laribus Augustis Imp(eratoris) Nervae Caesaris Traiani Aug(usti).

$\mathrm{Ci}$ si può chiedere d'altronde se si avvertisse una sostanziale differenza tra una dedica Victoriae Augustae, comiti dominorum principumq(ue) nostror(um) (A88) ed altra Victoriae Imp(eratoris) Caesaris Vespasiani Augusti, ${ }^{124}$ o tra due dediche, pressoché contemporanee ed entrambe della tribus Succusana, l'una Paci Augustae (A70), l'altra Paci aeternae domus Imp(eratoris) Vespasiani Caesaris Aug(usti) liberorumq(ue) eius. ${ }^{125}$

Ho il sospetto che così non fosse. Ritengo che, come della Victoria Augusta si poteva pensare e scrivere che era comes dominorum principumq(ue) nostror(um), così si potesse pensare, anche se non si scriveva, che Aesculapius, Apollo, Concordia, Diana e così via, in quanto Augusti / Augustae, stessero in analogo, privilegiato, rapporto con l'imperatore, e non tanto con la sovranità in generale, quanto con quell'imperatore che al momento occupava il trono. Forte di tanta divina assistenza, il principe, che non era dio, tuttavia agli occhi dei suoi sudditi non era più neppure un uomo. Si può dire in qualche modo che, come i suoi generali vincevano le battaglie, ma non celebravano il trionfo, ricevendo solo gli ornamenta triumphalia, così l'imperatore, non ancora diventato dio, ma tuttavia superata la condizione umana, non ricevesse ancora culto, ma fosse considerato già meritevole di ornamenta divina. Per certi gruppi di persone, in parte variabili secondo i tempi ed i luoghi (ed a Roma - come si è visto - eminentemente costituiti da schiavi e o liberti, particolarmente legati alla figura ed all'amministrazione imperiale), venerare alcune divinità chiamandole auguste poteva essere un modo per chieder loro di continuare ad essere garanti della riconosciuta sovrumanità del principe e per chiedere che esse, come assistevano l'imperatore nel suo operare a beneficio di tutti, così volessero estendere almeno in parte anche ad altri il loro potente aiuto.

\footnotetext{
${ }^{123}$ Contra Clauss 1999, op. cit. (n. 2), 280-285, che vede nell'uso del genitivo la volontà di istituire relazione con un particolare imperatore mentre la forma aggettivale connetterebbe il dio in generale con l'istituzione imperiale. Scettici sul punto, tra gli altri, già A.D. Nock, 'Studies in the Graeco-Roman Beliefs of the Empire', Journal of Hellenic Studies 45 (1925), 92 ss., Idem, Essays, op.cit. [n. 106], 41 ss.) e Fishwick 1991, op. cit. (n. 1), 446.

${ }^{124}$ CIL VI 198 cfr. 30712, 36747; Iscrizioni latine Napoli, I, 2000, op.cit. (n. 93), 58 nr. 5. Per una possibile dedica alla Vittoria di tre Augusti e due Cesari vedi ora G. L. Gregori, Zeitschrift für Papyrologie und Epigraphik 86 (1991), 285 s. nr. 2 (AE 1991, 274).

${ }^{125}$ CIL VI 200 cfr. 30712, 36747; Iscrizioni latine Napoli, I, 2000, op.cit. (n. 93), 58 ss. nr. 6.
} 
Così il culto delle divinità auguste a Roma ed altrove, pur con tutte le ambiguità che porta con sé, ${ }^{126}$ non presuppone in linea di principio l'idea di un imperatore-dio. Se mai, l'idea di un imperatore superuomo perché divinamente assistito al punto da essere compartecipe dei poteri degli dei. ${ }^{127}$

\author{
Roma, gennaio 2003
}

\begin{abstract}
${ }^{126}$ Sull'ambiguità, spesso intenzionale, come carattere da cui non si può prescindere nell'esame del fenomeno del cosiddetto culto imperiale, ambiguità favorita proprio perché "consentiva una ricezione dei messaggi ideologici flessibile e graduata, a più livelli, a seconda della cultura, delle tradizioni religiose e politiche, della sensibilità dei diversi soggetti in gioco": Letta 2002, op.cit. (n. 121), $628 \mathrm{~s}$.

${ }^{127}$ Solo al momento di consegnare questo scritto per la stampa ho potuto prendere visione di altri due recenti studi concernenti la questione qui trattata, uno in maniera generale, l'altro in modo specifico. Il primo è I. Gradel, Emperor Worship and Roman Religio (Oxford 2002), un libro che in qualche modo "sdrammatizza" l'opposizione uomo/dio in quanto l'autore ritiene che la divinità non sia un valore assoluto, bensì una qualità che sarebbe attribuita dal fedele sulla base di una riconosciuta detenzione di eccezionali (sovrumani) poteri. Onori divini sarebbero stati tributati già ad Augusto vivente in ambito privato e municipale; non invece nel culto pubblico; così in linea generale anche in seguito. Programmaticamente le divinità auguste non sono trattate. Una cosa sarebbe tuttavia attribuire epiteto di Augustus/Augusta ad un dio, che risulterebbe in tal modo vagamente collegato con l'imperatore, altra dire quello stesso dio Augusti, fatto che comporterebbe la nascita di una nuova divinità e la sua appropriazione da parte dell'imperatore come sua divinità tutelare (103-105). Dubito che questa distinzione, se anche poté esservi in origine, sia stata sempre mantenuta ed a tutti i livelli. Tutto il libro merita comunque un'approfondita discussione. Sulla presunta inesistenza di un culto del Genio di Augusto a Pompei ed altrove, vedi intanto i contributi di C. Letta citati sopra (n. 4). L'altro studio è l'articolo di A. Villaret, 'L'association de l'empereur et des dieux en Aquitaine. Son rôle dans la société et les mentalités', Aquitania 16 (1999), 127-151: esso consiste nella puntuale raccolta e nell'esame analitico delle testimonianze epigrafiche, da un lato di dedica congiunta all'imperatore ed a divinità (non a virtù) auguste. Abbiamo dunque un altro studio specifico sul tema. L'a. ritiene che non vi sia sostanziale differenza tra le due espressioni di culto, che costituirebbero piuttosto due diverse modalità di associazione degli dei all'imperatore, di cui diverrebbero protettrici particolari (il culto delle divinità auguste sarebbe stato prevalente al sud della provincia per influsso narbonese). Come a Roma, l'uso si estende dall'età augustea alla metà del $\mathrm{II}$ sec. e le divinità sono praticamente sempre Augustae e non Augusti. Notevoli invece le diversità per quanto riguarda gli dei prescelti (nella stragrande maggioranza interpretabili come gallo-romani o indigeni) e quanto ai dedicanti (essenzialmente notabili locali e peregrini; anche qualche donna; pochi gli schiavi ed i liberti). Non mancano testimonianze d'iniziativa pubblica, ma si tratta per lo più di culto privato, espressione, secondo l'a., «d'un sentiment religieux authentique qui allie "l'amour de l'Auguste" (Veyne) a la foi dans les dieux traditionelles de la cité, cooperation dont on attend des biens aussi vitaux que la santé, le salut, la fecondité, la prospérité». Altri studi analitici a carattere regionale potranno contribuire ad un'ulteriore precisazione del quadro.
\end{abstract}

